\title{
Closed-loop equilibrium strategies for general time-inconsistent optimal control problems
}

\author{
Tianxiao Wang* and Harry Zheng ${ }^{\dagger}$
}

\begin{abstract}
In this paper we introduce a general framework for time-inconsistent optimal control problems. We characterize the closed-loop equilibrium strategy in both the integral and pointwise forms with the newly developed methodology. We recover and improve the results of some well-known models, including the classical optimal control, Bjork et al. (2017), He and Jiang (2020), and Yong (2012) models, and reveal some interesting aspects that appear for the first time in the literature. We illustrate the usefulness of the model and the results by a number of examples in dynamic portfolio selection, including mean-variance with state-dependent risk aversion, investment/consumption with non-exponential discounting, and utility-deviation-risk with coupled terminal state and expected terminal state.
\end{abstract}

Keywords. time-inconsistent optimal control, random coefficients, closed-loop equilibrium strategy, BSPDEs, integral and pointwise characterizations

AMS Mathematics subject classification. Primary 93E20; Secondary 91B51, 60H10, 91G10.

\section{Introduction}

There has been extensive research and tremendous progress in the literature of dynamic optimization. One key feature of most papers is that the model is time-consistent in the sense that a decision deemed optimal yesterday is still optimal today and will continue to be optimal tomorrow. If this property is broken, then the problem is called time-inconsistent. Qualitative analysis on time-inconsistent behaviors of animals and human beings can be at least traced back to the works of Hume [16] in 1739 and of Smith [20] in 1759, see Palacios-Huerta [19] for an interesting survey.

Time-inconsistent problems are widely studied in economics and finance. Strotz [21] first showed some quantitative analysis on a deterministic Ramsey problem. There are mainly three different types of strategies to treat the time inconsistency in the literature: pre-commitment strategy, naive strategy and equilibrium strategy. The first one (the pre-committed agent) is derived from solving an optimization problem at initial time and then used throughout the whole time horizon, despite the fact that it may no longer be optimal at later time. The second one (the naive agent) is to pursue optimality at any time and ignore the resulting time inconsistency, which is quite riveting and has stimulated several recent works, see e.g. Eliaz and Splieger [7], He et al. [10], Huang et al. [15]. The last one (the sophisticated agent) is derived from solving a game-theoretic problem

${ }^{*}$ School of Mathematics, Sichuan University, Chengdu, PR China. Email: wtxiao2014@scu.edu.cn. Supported by the NSF of China under grants 11401404 and 11471231, the Science Development Project of Sichuan University under grant 2020SCUNL201.

${ }^{\dagger}$ Department of Mathematics, Imperial College, London, SW7 2AZ, UK. Email:h.zheng@imperial.ac.uk. Supported by the EPSRC (UK) standard research grant (EP/V008331/1). 
that ensures the strategies are consistent over the whole time period, which is a rational choice of a decision maker who is fully aware of time inconsistency.

There have been renewed interest and active research in recent literature on time-inconsistent model in the continuous time framework. Ekeland and Lazrak [4] and Ekeland and Pirvu [5] considered optimal consumption and investment under hyperbolic discounting in deterministic and stochastic models, and elegantly introduced a precise definition of the equilibrium concept. The idea is that, at every time instant $t$, the agent builds a coalition with his/her immediate selves in an infinitesimal time interval $[t, t+\varepsilon]$ and tries to minimize the cost functional, provided that the selves on $[t+\varepsilon, T]$ are agreed upon the desired equilibrium strategy. This is essentially a classical spike variation from the perspective of optimal control theory. Inspired by $[4,5]$, especially their definition of equilibrium strategy, Björk et al. [2] studied a general class of time-inconsistent optimal control problem in Markovian setting and derived, among other things, the so-called extended Hamilton-Jacobi-Bellman (HJB) system and the verification theorem. He and Jiang [11] introduced two new notions of equilibrium strategies, called the regular equilibria and strong equilibria, for time-inconsistent problems and their relations with the weak equilibria in [2]. Yong [28, 29] independently discussed a class of time-inconsistent optimal control problems with nonlinear state and cost functional by multi-person differential games approach, introduced a class of new equilibrium HJB equations and, unlike [2], started the investigation in continuous time setting, made partition on time intervals and used the theory of forward-backward stochastic differential equations (FBSDEs).

One common feature of aforementioned papers and almost all other papers in the literature is that the model coefficients and the objective functions are deterministic. The extension to nonMarkovian models with nonlinear state and cost is few and far between. The only papers on this topic the authors are aware of are Wang and Wu [23] and Hernández and Possamai [12]. In [23], the authors discussed a time-inconsistent problem with random coefficients and derived a family of backward stochastic partial differential equations (BSPDEs), called the stochastic equilibrium HJB equation, for the equilibrium value function by the multi-person differential games idea in $[28,29]$ and applied the results to a linear quadratic problem. Their model does not allow controls in the diffusion coefficient, nor initial state and expected terminal state in the objective function. In [12], the authors discussed a continuous time non-Markovian time-inconsistent problem in which the stochastic differential equation (SDE) for the state process depends on the trajectory of the state process and derived a system of backward stochastic differential equations (BSDEs) by the sub-game perfect Nash equilibrium and applied the results to a CRRA utility problem under the Markovian setting. They solved the problem in the weak sense (finding both the probability space and the equilibrium control) in contrast to the strong sense (finding the equilibrium control with a given probability space) of all other papers in the literature.

In this paper we introduce a general framework of time-inconsistent optimal control problems. We develop a new approach to characterizing the closed-loop equilibrium strategy in both integral and pointwise forms, recover and improve the well-known results in the existing papers, and reveal some interesting phenomena that appear for the first time in the literature. We now elaborate our contributions.

First, we propose a model setting that is more general than all those in the aforementioned literature (e.g. $[2,11,12,23,28]$ ). For example, the objective function in these papers is additively separable in the terminal state variable and its conditional expectation, which excludes the nonseparable utility-deviation-risk portfolio selection problem (Wong et al. [27]) that is a special case of our model. To the best knowledge of the authors, this is the first result in the literature on equilibrium strategies for general time-inconsistent and coupled utility deviation risk portfolio selection problems in continuous time framework. Our integral characterization of equilibrium 
strategy, with applications such as mean-variance problem with state-dependent risk aversion and investment-consumption problem with power utility and non-exponential discounting, allows the model coefficients to be random. These applications are beyond the scope of $[12,23]$. Our study opens a way of solving the following open problem mentioned in [2, Section 10]: "the present theory depends critically on the Markovian structure. It would be interesting to see what can be done without this assumption."

We develop a new methodology for solving the problem as follows: We first introduce a new definition of closed-loop equilibrium strategy, motivated by the randomness of the coefficients and the limitation in the relevant literature (Remark 2.1), then propose two terms $\Theta_{0}, \Xi$ (partially inspired by Wang and Yong [26]) that have the flow property and tackle the two kinds of time inconsistency caused by the cost functional (Remark 3.1), and finally establish the main results of the paper, i.e., the integral characterization (Theorem 3.1) and the pointwise counterpart (Theorems 4.1 and 4.2) for equilibrium strategy, by a new BSPDE system and a new PDE system, respectively. We make full use of the term $\bar{\Xi}$ (associated with equilibrium strategy $\bar{\Phi}$ ) in various characterizations to replace the equilibrium value function $(\mathrm{EVF})$. We may regard $\bar{\Xi}$ as a generalized $E V F$ which reduces to the EVF in a diagonal manner. Moreover, $\bar{\Xi}$ has the inherent flow property and natural connection with BSPDE system by the Itô-Kunita formula, while the EVF lacks these advantages. Some special forms of $\bar{\Xi}$ appear in $[28,29]$ that use the multi-person differential games idea (Subsection 5.4), a different approach compared with ours.

We now highlight some extension/improvement of our work on the relevant literature. First, to verify an equilibrium strategy in [2], one has to check the limit condition with arbitrary element in the Euclidean space, but He and Jiang [11, Footnote 6] showed that, for the mean-variance problem, such a requirement would be too strong to be satisfied and suggested the "support of distributions" of equilibrium state process, which still seems abstract and hard to verify. We directly use the value of equilibrium state process at time $t$, which is easy to verify and bypasses the above problematic issue. The new definition is also consistent with those in $[28,29]$. Second, a fundamental assumption in e.g. $[2,11]$ is that the closed-loop strategy and the model coefficients are right-continuous in time, needed for some limit argument by the Dominated Convergence Theorem (DCT). For a mean-variance portfolio selection model, such a requirement means that the riskless interest rate, the excess stock return, the stock volatility are either constant or right-continuous functions. We remove the continuity requirement by using a key result in Wang [25]. Third, when our cost functional reduces to that in e.g. $[2,11]$ with deterministic coefficients, we give a unified treatment on the necessary condition (see Lindensjö [17]) and the sufficient condition (see [2]) for equilibrium strategy, and our new PDE system essentially reduces to the HJB system in $[2,11,28]$ (see Section 5). We also recover the linear quadratic case in Wang [24] which relies on the FBSDEs approach.

Finally, we list some interesting facts arising from our study. First, due to the randomness of the coefficients, if we define the equilibrium strategy in terms of $\varepsilon>0$ like those in e.g. [2, 11], then the unaccountable property of $\varepsilon>0$ may cause the corresponding limit ill-posed. To avoid this issue, we use arbitrary sequence $\left\{\varepsilon_{n}\right\}$ tending to zero instead. Second, in the particular time-consistent Markovian setting (Subsection 5.1), we find that the equilibrium strategy, which is weaker than the optimal strategy, happens to be essentially equivalent to the optimal strategy and, to our surprise, that the HJB equation in the view of EVF can be obtained without the dynamic programming principle and the compact assumption of the control region (Remark 5.1). Third, we find two interesting phenomena for the investment/consumption model with CRRA utility and non-exponential discounting (Remark 3.7): One is that in the Markovian setting the time inconsistency caused by the discounting function does not affect the investment proportion of wealth, whereas in the non-Markovian case such coincidence disappears. The other is that for $\log$ 
utility the equilibrium consumption strategy is merely determined by the discounting functions and independent of other random coefficients, but for power utility the same feature does not hold.

The rest of the paper is organized as follows. In Section 2 we describe the time-inconsistent model with random coefficients and define the closed-loop equilibrium strategy. In Section 3 we give the integral characterization for equilibrium strategy (Theorem 3.1) and apply it to solve meanvariance and investment/consumption problems with random coefficients. In Section 4 we discuss the Markovian case and give two pointwise characterizations for equilibrium strategy (Theorems 4.1 and 4.2). In Section 5 we compare and recover the results of some well-known models in the Markovian setting, including classical optimal control, Bjork et al. [2], He and Jiang [11], and Yong [28] models. Section 6 concludes. The appendix contains the proofs of all theorems.

\section{Problem formulation}

Suppose $\left(\Omega, \mathcal{F}, \mathbb{P},\left\{\mathcal{F}_{t}\right\}_{t \geq 0}\right)$ is a filtered complete probability space, $\{W(t), t \geq 0\}$ is a $\mathbb{F}:=\left\{\mathcal{F}_{t}\right\}_{t \geq 0^{-}}$ adapted one-dimensional Brownian motion. For $p>1$, denote by $L_{\mathcal{F}_{t}}^{2}\left(\Omega ; \mathbb{R}^{n}\right)$ the space of $\mathcal{F}_{t^{-}}$ measurable random variables $\xi$ such that $\mathbb{E}|\xi|^{2}<\infty, L_{\mathbb{F}}^{p}\left(\Omega ; C\left([0, T] ; \mathbb{R}^{n}\right)\right)$ the space of all $\mathbb{F}$-adapted, $\mathbb{R}^{n}$-valued, continuous processes $X$ such that $\mathbb{E}\left(\sup _{r \in[0, T]}|X(r)|^{2}\right)^{p}<\infty$ and $L_{\mathbb{F}}^{p}\left(\Omega ; L^{2}\left(0, T ; \mathbb{R}^{n}\right)\right)$ the space of all $\mathbb{F}$-adapted, $\mathbb{R}^{n}$-valued processes $X$ such that $\mathbb{E}\left(\int_{0}^{T}|X(r)|^{2} d r\right)^{\frac{p}{2}}<\infty$. If $p=2$, then $L_{\mathbb{F}}^{2}\left(0, T ; \mathbb{R}^{n}\right) \equiv L_{\mathbb{F}}^{2}\left(\Omega ; L^{2}\left(0, T ; \mathbb{R}^{n}\right)\right)$. Given $r \in[0, T], y \in \mathbb{R}^{n}, l \in \mathbb{N}$, mapping $f: \Omega \times[0, T] \times$ $\mathbb{R}^{n} \rightarrow \mathbb{R}^{l}$, denote by $\partial_{y} f(r, y) \in \mathbb{R}^{l \times n}$ and $\partial_{y y} f(r, y) \in \mathbb{R}^{l \times(n \times n)}$ the first and second order partial derivatives of $f$ with respect to $y$ and evaluated at $(r, y)$. Note that for $a, b \in \mathbb{R}^{n}, a^{\top} \partial_{y y} f(r, y) b \in \mathbb{R}^{l}$. To shorten notations, we write $C^{1,2} \equiv C^{1,2}\left([0, T] \times \mathbb{R}^{n} ; \mathbb{R}\right), C_{n}^{1,2} \equiv C^{1,2}\left([0, T] \times \mathbb{R}^{n} ; \mathbb{R}^{n}\right)$. Similar notations are used for other spaces, e.g. $C^{0,1}$ and $C_{n}^{0,1}$.

For $n, m \in \mathbb{N}$, we introduce the following assumption.

(H1) Suppose $b, \sigma: \Omega \times[0, T] \times \mathbb{R}^{n} \times \mathbb{R}^{m} \rightarrow \mathbb{R}^{n}, g: \Omega \times[0, T] \times[0, T] \times \mathbb{R}^{n} \times \mathbb{R}^{n} \times \mathbb{R}^{m} \rightarrow \mathbb{R}$, $h: \Omega \times[0, T] \times \mathbb{R}^{n} \times \mathbb{R}^{n} \times \mathbb{R}^{n} \rightarrow \mathbb{R}$ are measurable processes such that for $(t, \gamma, x, u, y) \in[0, T] \times \mathbb{R}^{n} \times$ $\mathbb{R}^{n} \times \mathbb{R}^{m} \times \mathbb{R}^{n}, b(\cdot, x, u), \sigma(\cdot, x, u), g(t, \cdot, \gamma, x, u)$ are $\mathcal{F}$-progressively measurable, and $\partial_{y} h(t, \gamma, x, y)$ exits.

Under (H1), given $x \in \mathbb{R}^{n}$, we consider the following SDE:

$$
\left\{\begin{array}{l}
d X(s)=b(s, X(s), u(s)) d s+\sigma(s, X(s), u(s)) d W(s), \quad s \in[0, T], \\
X(0)=x
\end{array}\right.
$$

where $X(\cdot)$ is the state process and $u(\cdot)$ the control process that ensures (2.1) admits a unique strong solution. For any $t \in[0, T)$, and $\mathcal{F}_{t}$-measurable random variable $X(t)$, we consider the cost functional

$$
J(u(\cdot) ; t, X(t))=\mathbb{E}_{t}\left[\int_{t}^{T} g(t, s, X(t), X(s), u(s)) d s+h\left(t, X(t), X(T), \mathbb{E}_{t} X(T)\right)\right],
$$

where $\mathbb{E}_{t}$ is the conditional expectation given $\mathcal{F}_{t}$. The pre-committed (or naive) strategy with initial condition $(t, X(t))$ is to find a $\bar{u}(\cdot)$ that minimizes $J(u(\cdot) ; t, X(t))$ in the essentially infimum (essinf) sense over all controls $u(\cdot)$ that make $(2.2)$ well defined. However, in this paper we seek the equilibrium strategy. In the following, instead of control $u(\cdot)$, we consider feedback (closed-loop) strategy $\Phi$ which is a measurable mapping $\Phi: \Omega \times[0, T] \times \mathbb{R}^{n} \rightarrow \mathbb{R}^{m}$ such that for any $t \in[0, T]$, $x \in \mathbb{R}^{n}, \Phi(t, x)$ is $\mathcal{F}_{t}$-measurable. Given $(t, s) \in[0, T] \times[0, T],(\gamma, x) \in \mathbb{R}^{n} \times \mathbb{R}^{n}$, let

$$
f^{\Phi}(s, x):=f(s, x, \Phi(s, x)), \quad f:=b, \sigma, g^{\Phi}(t, s, \gamma, x):=g(t, s, \gamma, x, \Phi(s, x)) .
$$


Definition 2.1 A closed-loop strategy $\Phi$ is called admissible if for any $x \in \mathbb{R}$,

$$
\left\{\begin{array}{l}
d \mathscr{X}(s)=b^{\Phi}(s, \mathscr{X}(s)) d s+\sigma^{\Phi}(s, \mathscr{X}(s)) d W(s), \quad s \in[0, T], \\
\mathscr{X}(0)=x,
\end{array}\right.
$$

admits a unique strong solution $\mathscr{X}(\cdot) \equiv \mathscr{X}^{\Phi, 0, x}(\cdot)$ such that for any $t \in[0, T)$,

$$
\mathbb{E}_{t}\left[\int_{t}^{T}\left|g^{\Phi}(t, s, \mathscr{X}(t), \mathscr{X}(s))\right| d s+\left|h\left(t, \mathscr{X}(t), \mathscr{X}(T), \mathbb{E}_{t} \mathscr{X}(T)\right)\right|+|\mathscr{X}(T)|\right]<\infty .
$$

The set of all admissible strategies is denoted by $\mathscr{S}_{\text {ad }}$.

In the following, when it is necessary to emphasize the dependence of $\mathscr{X}(\cdot)$ on $(\Phi, 0, x)$, we use $\mathscr{X}^{\Phi, 0, x}(\cdot)$, otherwise, we simply write it as $\mathscr{X}(\cdot)$. Condition $(2.5)$ can be easily checked if $g^{\Phi}$, $h$, and $\mathscr{X}(\cdot)$ satisfy proper growth and integrability conditions and is mainly used to keep the following cost functional, derived from $(2.2)$ by replacing $u(\cdot)$ with $\Phi$, well-defined,

$$
J(\Phi ; t, \mathscr{X}(t)):=\mathbb{E}_{t}\left[\int_{t}^{T} g^{\Phi}(t, s, \mathscr{X}(t), \mathscr{X}(s)) d s+h\left(t, \mathscr{X}(t), \mathscr{X}(T), \mathbb{E}_{t} \mathscr{X}(T)\right)\right] .
$$

For any $\bar{\Phi}, \Phi^{\prime} \in \mathscr{S}_{a d}, t \in[0, T), \varepsilon>0$ small enough such that $t+\varepsilon \leq T$, define

$$
\Phi^{\varepsilon}(s, x)=\bar{\Phi}(s, x) I_{[0, t) \cup[t+\varepsilon, T]}(s)+\Phi^{\prime}(s, x) I_{[t, t+\varepsilon)}(s) .
$$

For a given $\bar{\Phi} \in \mathscr{S}_{a d}$, we define $\overline{\mathscr{S}}_{a d}$ the set of all closed-loop strategies $\Phi^{\prime}$ such that $\Phi^{\varepsilon} \in \mathscr{S}_{a d}$. The set $\overline{\mathscr{S}}_{a d}$ is implicit and abstract, and is specified with explicit form in various special settings. Let $\mathscr{X}^{\varepsilon}(\cdot)$ be the strong solution of $(2.4)$ with $\Phi^{\varepsilon}$. By uniqueness, $\mathscr{X}^{\varepsilon}(\cdot)=\mathscr{X}(\cdot)$ on $[0, t]$. We now introduce the notion of closed-loop equilibrium strategy.

Definition 2.2 A strategy $\bar{\Phi} \in \mathscr{S}_{\text {ad }}$ is called a closed-loop equilibrium strategy if for any $t \in[0, T)$, any $\Phi^{\prime} \in \overline{\mathscr{S}}_{\text {ad }}$, and any sequence $\left\{\varepsilon_{n}\right\}$ tending to zero, we have

$$
\lim _{\varepsilon_{n} \rightarrow 0} \frac{J\left(\Phi^{\varepsilon_{n}} ; t, \overline{\mathscr{X}}(t)\right)-J(\bar{\Phi} ; t, \overline{\mathscr{X}}(t))}{\varepsilon_{n}} \geq 0,
$$

where $\overline{\mathscr{X}}(\cdot)$ is the strong solution of (2.4) with $(\bar{\Phi}, 0, x)$. A function $V:[0, T] \times \mathbb{R}^{n} \rightarrow \mathbb{R}^{n}$ is called the equilibrium value function $(E V F)$ if $V(t, \overline{\mathscr{X}}(t))=J(\bar{\Phi} ; t, \overline{\mathscr{X}}(t))$ for all $t \in[0, T], x \in \mathbb{R}^{n}$.

Note that in (2.8) the limit is taken with any sequence $\left\{\varepsilon_{n}\right\}$ tending to 0 , not $\varepsilon \in \mathbb{R}$ tending to 0 . In fact, in our non-Markovian framework, the difference quotient in (2.8) with $\varepsilon_{n}$ replaced by $\varepsilon$ is well-defined in a full measure with respect to $\omega$. Due to the uncountability property of $\varepsilon>0$, the limit in (2.8) may not be well-defined and this is the reason of using $\left\{\varepsilon_{n}\right\}$ instead.

Remark 2.1 Given $\Phi \in \mathscr{S}_{a d}, t \in[0, T)$, and $\mathcal{F}_{t}$-measurable random variable $\xi$, let $\mathcal{J}(\Phi ; t, y)$ be the restriction of $J(\Phi ; t, \xi)$ on $\mathbb{R}^{n}$. Under proper conditions, one has $J(\Phi ; t, \xi)=\left.\mathcal{J}(\Phi ; t, y)\right|_{y=\xi}$ a.s. (see Appendix A.4), which enables us to present another equivalent statement of (2.8) by $\mathcal{J}$. Note that $\mathcal{J}$ still depends on $\omega$ due to the randomness of $b, \sigma, g, h$, but is deterministic in the Markovian setting. In some literature, e.g. [2, 11], the authors replace $J, \xi$ in $(2.8)$ by $\mathcal{J}, y$, respectively, where $y$ is in a subset of $\mathbb{R}^{n}$ or even $\mathbb{R}^{n}$ itself. Here we adopt the idea from [24, Definition 2.3], [28, Definition 4.1]. Our version avoids the problematic issue of [2] pointed in [11, Footnote 6] and also reduces to the classical notions in specific important models (Subsection 5.1). 


\section{$3 \quad$ Integral characterizations with random coefficients}

Given $(\tau, \gamma) \in[0, T) \times \mathbb{R}^{n}, \Phi \in \mathscr{S}_{\text {ad }}$, inspired by the backward PDEs in [26, Theorem 1.2], we introduce the following BSPDE system: for $t \in[0, T], \gamma, y \in \mathbb{R}^{n}$,

$$
\left\{\begin{array}{c}
\Lambda(t, y)=y+\int_{t}^{T}\left[(\mathscr{A} \Lambda(s, \cdot))^{\Phi}(y)+\partial_{y} \Gamma(s, y) \sigma^{\Phi}(s, y)\right] d s-\int_{t}^{T} \Gamma(s, y) d W(s) \\
\Xi(\tau, t, \gamma, y)=h(\tau, \gamma, y, \Lambda(\tau, \gamma))+\int_{t}^{T}\left[(\mathscr{A} \Xi(\tau, s, \gamma, \cdot))^{\Phi}(y)+g^{\Phi}(\tau, s, \gamma, y)\right. \\
\left.\quad+\partial_{y} \Delta(\tau, s, \gamma, y) \sigma^{\Phi}(s, y)\right] d s-\int_{t}^{T} \Delta(\tau, s, \gamma, y) d W(s)
\end{array}\right.
$$

where $\mathscr{A}$ is an infinitesimal generator, defined by, for a $\mathbb{R}^{n}$-valued (or $\mathbb{R}$-valued) function $g$,

$$
(\mathscr{A} g(r, \cdot))^{\Phi}(x):=\partial_{x} g(r, x) b^{\Phi}(r, x)+\frac{1}{2} \sigma^{\Phi}(r, x)^{\top} \partial_{x x} g(r, x) \sigma^{\Phi}(r, x) .
$$

In this paper, we are interested in the classical solution of (3.1), see e.g. Hu et al. [13]. Given $\bar{\Phi}$, $\Phi^{\prime} \in \mathscr{S}_{a d}$, for any $t, r \in[0, T), \gamma, x \in \mathbb{R}^{n}$, define

$$
\begin{aligned}
& \delta b(r, x):=b^{\Phi^{\prime}}(r, x)-b^{\bar{\Phi}}(r, x), \quad \delta \sigma(r, x):=\sigma^{\Phi^{\prime}}(r, x)-\sigma^{\bar{\Phi}}(r, x), \\
& \delta g(t, r, \gamma, x):=g^{\Phi^{\prime}}(t, r, \gamma, x)-g^{\bar{\Phi}}(t, r, \gamma, x), \\
& \delta \mathscr{G}_{1}(r, x):=(\mathscr{A} \bar{\Lambda}(r, \cdot))^{\Phi^{\prime}}(x)-(\mathscr{A} \bar{\Lambda}(r, \cdot))^{\bar{\Phi}}(x)+\partial_{x} \bar{\Gamma}(r, x)[\delta \sigma(r, x)], \\
& \delta \mathscr{G}_{2}(t, r, \gamma, x):=(\mathscr{A} \bar{\Xi}(t, r, \gamma, \cdot))^{\Phi^{\prime}}(x)-(\mathscr{A} \bar{\Xi}(t, r, \gamma, \cdot))^{\bar{\Phi}}(x)+\partial_{x} \bar{\Delta}(t, r, \gamma, x)[\delta \sigma(r, x)], \\
& H(t, \varepsilon):=\left.\int_{0}^{1} \partial_{y} h\left(t, \overline{\mathscr{X}}(t), \mathscr{X}^{\varepsilon}(T), y\right)\right|_{y=(1-\theta) \mathbb{E}_{t} \overline{\mathscr{X}}(T)+\theta \mathbb{E}_{t} \mathscr{X}^{\varepsilon}(T)} d \theta, \\
& \mathcal{S}^{\Phi^{\varepsilon}, \bar{\Phi}}(t, \varepsilon):=\frac{1}{\varepsilon} \mathbb{E}_{t} \int_{t}^{t+\varepsilon}\left(\delta g\left(t, r, \overline{\mathscr{X}}(t), \mathscr{X}^{\varepsilon}(r)\right)\right. \\
& \left.+\left[\mathbb{E}_{t} H(t, \varepsilon)\right] \delta \mathscr{G}_{1}\left(r, \mathscr{X}^{\varepsilon}(r)\right)+\delta \mathscr{G}_{2}\left(t, r, \overline{\mathscr{X}}(t), \mathscr{X}^{\varepsilon}(r)\right)\right) d r,
\end{aligned}
$$

where $\mathscr{X}^{\varepsilon}(\cdot)$ is a solution of $(2.4)$ associated with $\Phi^{\varepsilon}$ and $(\bar{\Lambda}, \bar{\Gamma}),(\bar{\Xi}, \bar{\Delta})$ satisfy $(3.1)$ with $\bar{\Phi}$.

(H2) Suppose (H1) holds true, $\bar{\Phi} \in \mathscr{S}_{\text {ad }}$ such that (3.1) admits classical solutions $(\bar{\Lambda}, \bar{\Gamma})$ and $(\bar{\Xi}, \bar{\Delta})$ satisfying

$$
\begin{aligned}
& \mathbb{E}_{t}\left[\left(\left.\int_{t}^{T}\left|\bar{\Gamma}(r, y)+\partial_{x} \bar{\Lambda}(r, y) \sigma^{\Phi}(r, y)\right|^{2}\right|_{y=\mathscr{X}(r)} d r\right)^{\frac{1}{2}}\right]<\infty, \\
& \mathbb{E}_{t}\left[\left(\left.\int_{t}^{T}\left|\bar{\Delta}(t, r, \mathscr{X}(t), z)+\partial_{z} \bar{\Xi}(t, r, \mathscr{X}(t), z) \sigma^{\Phi}(r, z)\right|^{2}\right|_{z=\mathscr{X}(r)} d r\right)^{\frac{1}{2}}\right]<\infty,
\end{aligned}
$$

with $(\overline{\mathscr{X}}(\cdot), \bar{\Phi})$. Moreover, there exists a closed-loop strategy $\Phi^{\prime}$ such that $\Phi^{\varepsilon}$ in $(2.7)$ is admissible and (3.4) holds corresponding to $\left(\mathscr{X}^{\varepsilon}(\cdot), \Phi^{\varepsilon}\right)$. We assume $t \in[0, T)$ and $\varepsilon \in(0, T-t)$. In addition,

$$
\begin{gathered}
\mathbb{E}_{t}\left[\int_{t}^{t+\varepsilon}\left(\left|\delta \mathscr{G}_{1}\left(r, \mathscr{X}^{\varepsilon}(r)\right)\right|+\left|\delta \mathscr{G}_{2}\left(t, r, \overline{\mathscr{X}}(t), \mathscr{X}^{\varepsilon}(r)\right)\right|+\left|g^{\bar{\Phi}}\left(t, r, \overline{\mathscr{X}}(t), \mathscr{X}^{\varepsilon}(r)\right)\right|\right) d r\right. \\
\left.+|H(t, \varepsilon)|+\left|h\left(t, \overline{\mathscr{X}}(t), \mathscr{X}^{\varepsilon}(T), \mathbb{E}_{t} \overline{\mathscr{X}}(T)\right)\right|\right]<\infty .
\end{gathered}
$$

We can now state the first main result of the paper: the integral characterization for equilibrium strategy. 
Theorem 3.1 Suppose (H2) holds with the given $\bar{\Phi}, \Phi^{\varepsilon} \in \mathscr{S}_{\text {ad }}$. Then

$$
\frac{J\left(\Phi^{\varepsilon} ; t, \overline{\mathscr{X}}(t)\right)-J(\bar{\Phi} ; t, \overline{\mathscr{X}}(t))}{\varepsilon}=\mathcal{S}^{\Phi^{\varepsilon}, \bar{\Phi}}(t, \varepsilon) .
$$

Furthermore, if (H2) holds for any $\Phi^{\prime} \in \overline{\mathscr{S}}_{\text {ad }}$, then $\bar{\Phi}$ is an equilibrium strategy if and only if

$$
\lim _{\varepsilon_{n} \rightarrow 0} \mathcal{S}^{\Phi^{\varepsilon_{n}}, \bar{\Phi}}\left(t, \varepsilon_{n}\right) \geq 0
$$

Proof. See Section A.1.

Remark 3.1 To address the time inconsistency of $J(u(\cdot) ; t, \mathscr{X}(t))$ due to the dependence of $g, h$ on $t, \mathscr{X}(t), \mathbb{E}_{t} \mathscr{X}(T)$, we introduce an alternative "time consistent" version which closely relates to $J$. Specifically, for any $x \in \mathbb{R}^{n}, \Phi \in \mathscr{S}_{a d}, 0 \leq t \leq r \leq T, \mathscr{X}(\cdot)=\mathscr{X}^{\Phi, 0, x}(\cdot), \eta:=\mathscr{X}(t), \xi:=\mathscr{X}(r)$, we propose the following term

$$
\Theta_{0}(t, r, \eta, \xi):=\mathbb{E}_{r}\left[\int_{r}^{T} g^{\Phi}(t, s, \eta, \mathscr{X}(s)) d s+h\left(t, \eta, \mathscr{X}(T), \mathbb{E}_{t} \mathscr{X}(T)\right)\right]
$$

which is well-defined and useful in the proof of Theorem 3.1. It is more general than $J$ in the sense that $\Theta_{0}(t, t, \eta, \eta)=J(\Phi ; t, \eta)$ if $t=r$. More importantly, for any $r \geq t$,

$$
\Theta_{0}(t, t, \mathscr{X}(t), \mathscr{X}(t))=\mathbb{E}_{t}\left[\Theta_{0}(t, r, \mathscr{X}(t), \mathscr{X}(r))+\int_{t}^{r} g^{\Phi}(t, s, \mathscr{X}(t), \mathscr{X}(s)) d s\right] .
$$

The above relation is referred as the flow property which is closely relate to the classical dynamic programming principle. In some sense, such a property treats the time inconsistency caused by $g$.

We also rely on $\Xi$ in (3.1) to tackle the time inconsistency caused by $\mathbb{E}_{t} \mathscr{X}(T)$. The term $\Xi$ connects $\Theta_{0}$ via $H(t, \varepsilon)$ in (A.2). Unlike $\Theta_{0}$, it is defined on Euclidean vector (rather than random variable) which makes the well-known Itô-Kunita formula applicable. Note that $\Xi$ also satisfies the flow property in the sense of $(3.8)$, and $J(\Phi ; t, \mathscr{X}(t))=\Xi(t, t, \mathscr{X}(t), \mathscr{X}(t))$. If $\bar{\Phi}$ is the equilibrium strategy, then in terms of Definition $2.2, \bar{\Xi}(t, t, x, x)$, with $(t, x) \in[0, T] \times \mathbb{R}^{n}$, is the EVF, and $\bar{\Xi}$ may be regarded as a generalized $E V F$ that reduces to the EVF in a diagonal manner. Its usefulness is demonstrated in Theorem 3.1 and the following sections. Some special forms of $\Xi$ appear in [2, (6.4-6.5)] and [28, Section 5], but their roles are not explored.

Remark 3.2 To the best knowledge of the authors, the conclusions in Theorem 3.1 appear for the first time even in the special framework (e.g. [2, 11, 12]). Motivated by $(2.8)$, we derive an explicit equality for the difference of perturbed cost and equilibrium cost, which immediately leads to the integral characterization for equilibrium strategy with random coefficients. For later use, we point out a special case with $h$ being free of $\mathbb{E}_{t} \mathscr{X}(T)$. Then $H(t, \varepsilon)=0$ in (3.3), which makes the term $\delta \mathscr{G}_{1}\left(r, \mathscr{X}^{\varepsilon}(r)\right)$ irrelevant in (3.6). Hence $\Lambda, \Gamma$ in BSPDE (3.1) can be removed.

In the next two subsections, we apply Theorem 3.1 to two special settings with random coefficients, while most existing papers (e.g. [1, 2, 3, 5, 14, 18, 28]) assume deterministic coefficients. For these models, the BSPDE system (3.1) can be reduced to the BSDE systems, some of which appear for the first time in the literature. Denote by $\mathscr{H}_{1}:=L_{\mathbb{F}}^{2}(\Omega ; C([0, T] ; \mathbb{R})) \times L_{\mathbb{F}}^{2}(0, T ; \mathbb{R})$. 


\subsection{Mean-variance with state-dependent risk aversion}

Suppose that $A(\cdot), B(\cdot), D(\cdot)$ are bounded $\mathbb{F}$-adapted processes, $x \in \mathbb{R}$. Given a proper strategy $\Phi$, we consider a one-dimensional controlled SDE:

$$
\begin{aligned}
& d \mathscr{X}(s)=[A(s) \mathscr{X}(s)+B(s) \Phi(s, \mathscr{X}(s))] d s+D(s) \Phi(s, \mathscr{X}(s)) d W(s), s \in[0, T], \\
& \mathscr{X}(0)=x .
\end{aligned}
$$

For any $t \in[0, T)$, the cost functional at time $t$ is given by

$$
J(\Phi ; t, \mathscr{X}(t))=\mathbb{E}_{t}\left[\mathscr{X}(T)^{2}\right]-\left(\mathbb{E}_{t}[\mathscr{X}(T)]\right)^{2}-\mathcal{R}(\mathscr{X}(t)) \mathbb{E}_{t}[\mathscr{X}(T)],
$$

where for a constant $\mathcal{R}>0, \mathcal{R}(x)=\mathcal{R}$ or $\mathcal{R}(x)=\mathcal{R} x$ for all $x$. SDE (3.9) can be interpreted as a wealth equation with $\mathscr{X}(\cdot)$ the wealth, $A(\cdot)$ the riskless interest rate, $B(\cdot)$ the excess stock return, $D(\cdot)$ the stock volatility, and $\Phi(\cdot, \mathscr{X}(\cdot))$ the amount of money invested in the risky stock. This is a continuous time time-inconsistent mean-variance problem.

The model (3.9) and the objective (3.10) can be written as (2.1) and (2.2) with

$$
\left\{\begin{array}{l}
b(s, x, u)=A(s) x+B(s) u, \quad \sigma(s, x, u)=D(s) u, \\
g(t, s, \gamma, x, u)=0, \quad h(t, \gamma, x, y)=x^{2}-y^{2}-\mathcal{R}(\gamma) x .
\end{array}\right.
$$

Due to the linear-quadratic structure, also the terminal condition

$$
\bar{\Lambda}(T, y)=y, \bar{\Xi}(\tau, T, \gamma, y)=h(\tau, \gamma, y, \bar{\Lambda}(\tau, \gamma))=y^{2}-\bar{\Lambda}(\tau, \gamma)^{2}-\mathcal{R}(\gamma) y
$$

we propose the following ansatz form on all admissible strategies:

$$
\bar{\Phi}(s, y)=\bar{\phi}(s) y+\bar{\varphi}(s), \quad \Phi^{\prime}(s, y)=\bar{\phi}(s) y+\bar{\varphi}(s)+v, \quad s \in[0, T], \quad y \in \mathbb{R},
$$

with any $v \in \mathbb{R}$ and some proper processes $(\bar{\phi}, \bar{\varphi})$, and the BSPDE solution $(\bar{\Lambda}, \bar{\Gamma}),(\bar{\Xi}, \bar{\Delta})$ to $(3.1)$, associated with $\bar{\Phi}$, given by

$$
\left\{\begin{array}{l}
\bar{\Lambda}(s, y):=\lambda_{0}(s) y+\lambda_{1}(s), \quad \bar{\Gamma}(s, y):=\widetilde{\lambda}_{0}(s) y+\widetilde{\lambda}_{1}(s), \\
\bar{\Xi}(\tau, t, \gamma, y):=\lambda_{2}(t) y^{2}+\left(\lambda_{3}(t)-\mathcal{R}(\gamma) \lambda_{0}(t)\right) y+\lambda_{4}(t)-\bar{\Lambda}(\tau, \gamma)^{2}, \\
\bar{\Delta}(\tau, t, \gamma, y):=\widetilde{\lambda}_{2}(t) y^{2}+\left(\widetilde{\lambda}_{3}(t)-\mathcal{R}(\gamma) \widetilde{\lambda}_{0}(t)\right) y+\widetilde{\lambda}_{4}(t),
\end{array}\right.
$$

where $\left(\lambda_{i}, \widetilde{\lambda}_{i}\right)$ are to be determined. Substituting $(\bar{\Lambda}, \bar{\Gamma})$ and $(\bar{\Xi}, \bar{\Delta})$ into $(3.1)$, collecting terms, we can easily obtain the following system of BSDEs: for $t \in[0, T]$,

$$
\left\{\begin{array}{l}
\lambda_{0}(t)=1+\int_{t}^{T}\left[\lambda_{0} A+\lambda_{0} B \bar{\phi}+\widetilde{\lambda}_{0} D \bar{\phi}\right](s) d s-\int_{t}^{T} \widetilde{\lambda}_{0}(s) d W(s), \\
\lambda_{1}(t)=\int_{t}^{T}\left[\lambda_{0} B \bar{\varphi}+\widetilde{\lambda}_{0} D \bar{\varphi}\right](s) d s-\int_{t}^{T} \widetilde{\lambda}_{1}(s) d W(s), \\
\lambda_{2}(t)=1+\int_{t}^{T}\left[\lambda_{2} D^{2} \bar{\phi}^{2}+2 \lambda_{2} A+2 \lambda_{2} B \bar{\phi}+2 \widetilde{\lambda}_{2} D \bar{\phi}\right](s) d s-\int_{t}^{T} \widetilde{\lambda}_{2}(s) d W(s), \\
\lambda_{3}(t)=\int_{t}^{T}\left[\lambda_{3} A+\lambda_{3} B \bar{\phi}+\widetilde{\lambda}_{3} D \bar{\phi}+2 \lambda_{2} D^{2} \bar{\phi} \bar{\varphi}+2 \lambda_{2} B \bar{\varphi}+2 \widetilde{\lambda}_{2} D \bar{\varphi}\right](s) d s-\int_{t}^{T} \widetilde{\lambda}_{3}(s) d W(s), \\
\lambda_{4}(t)=\int_{t}^{T}\left[\lambda_{2} D^{2} \bar{\varphi}^{2}+\lambda_{3} B \bar{\varphi}+\widetilde{\lambda}_{3} D \bar{\varphi}\right](s) d s-\int_{t}^{T} \widetilde{\lambda}_{4}(s) d W(s) .
\end{array}\right.
$$


This greatly simplifies the problem as one only needs to solve BSDEs, not BSPDEs.

(H3) Suppose for any $p>2,(\bar{\phi}, \bar{\varphi}) \in L_{\mathbb{F}}^{p}\left(\Omega ; L^{2}\left(0, T ; \mathbb{R}^{2}\right)\right)$ and

(i) for any $v \in \mathbb{R}, \operatorname{SDE}(3.9)$ admits a unique solution $\overline{\mathscr{X}}(\cdot), \mathscr{X}^{\varepsilon}(\cdot) \in L_{\mathbb{F}}^{p}(\Omega ; C([0, T] ; \mathbb{R}))$ associated with $\bar{\Phi}, \Phi^{\varepsilon}$, respectively.

(ii) BSDE system (3.12) is solvable such that $\left(\lambda_{i}, \widetilde{\lambda}_{i}\right) \in \mathscr{H}_{1}, i=0,1,2,3,4$.

(iii) Some limiting condition in the sense of (A.5), (A.6) hold.

Under (H3) and (3.11), $\bar{\Phi} \in \mathscr{S}_{\text {ad }}$. Let $\overline{\mathscr{M}}$ be the set of $\Phi^{\prime}$ in (3.11) such that $(\bar{\phi}, \bar{\varphi})$ satisfy (H3). Clearly, $\overline{\mathscr{M}} \subset \overline{\mathscr{S}}_{a d}$. In this subsection, the equilibrium strategy is defined with $\overline{\mathscr{S}}_{\text {ad }}$ replaced by $\overline{\mathscr{M}}$. Given functions $f, g$, let $\mathscr{K}_{f, g}(t):=B(t) f(t)+D(t) g(t)$ for $t \in[0, T]$.

Theorem 3.2 Assume $A(\cdot), B(\cdot), D(\cdot)$ are bounded $\mathbb{F}$-adapted processes, $x \in \mathbb{R}$.

(i) Suppose $\mathcal{R}(x)=\mathcal{R}$, a constant, and there exists $(\bar{\phi}, \bar{\varphi})$ satisfying (H3). Then $\bar{\Phi}$ is a closed-loop equilibrium strategy if and only if for $t \in[0, T), D^{2}(t) \lambda_{2}(t) \geq 0$ and

$$
\begin{aligned}
& \mathscr{K}_{\lambda_{2}, \widetilde{\lambda}_{2}}(t)-\lambda_{0}(t) \mathscr{K}_{\lambda_{0}, \widetilde{\lambda}_{0}}(t)+D^{2}(t) \bar{\phi}(t) \lambda_{2}(t)=0, \\
& \mathscr{K}_{\lambda_{3}, \widetilde{\lambda}_{3}}(t)-\left(\mathcal{R}+2 \lambda_{1}(t)\right) \mathscr{K}_{\lambda_{0}, \widetilde{\lambda}_{0}}(t)+2 D^{2}(t) \bar{\varphi}(t) \lambda_{2}(t)=0 .
\end{aligned}
$$

(ii) Suppose $\mathcal{R}(x)=\mathcal{R} x$ and there exists $(\bar{\phi}, 0)$ satisfying (H3). Then $\bar{\Phi}$ is a closed-loop equilibrium strategy if and only if for $t \in[0, T], D^{2}(t) \lambda_{2}(t) \geq 0$ and

$$
\mathscr{K}_{\lambda_{2}, \tilde{\lambda}_{2}}(t)-\lambda_{0}(t) \mathscr{K}_{\lambda_{0}, \widetilde{\lambda}_{0}}(t)-\frac{1}{2} \mathcal{R} \mathscr{K}_{\lambda_{0}, \widetilde{\lambda}_{0}}(t)+D^{2}(t) \bar{\phi}(t) \lambda_{2}(t)=0 .
$$

Proof. See Section A.2.

Remark 3.3 When the coefficients are deterministic, then $\widetilde{\lambda}_{i}=0, i=0,1,2,3,4$. If $\mathcal{R}(x)=\mathcal{R}$, $(\bar{\phi}, \bar{\varphi}) \in L^{2}\left(0, T ; \mathbb{R}^{2}\right)$, then (H3) automatically holds. We have $\bar{\Phi}=\bar{\phi} x+\bar{\varphi}$ is a closed-loop equilibrium strategy if and only if for $t \in[0, T), D^{2}(t) \lambda_{2}(t) \geq 0$,

$$
\begin{aligned}
& {\left[\lambda_{2}(t)-\lambda_{0}^{2}(t)\right] B(t)+D^{2}(t) \bar{\phi}(t) \lambda_{2}(t)=0,} \\
& B(t)\left[\lambda_{3}(t)-\left(\mathcal{R}+2 \lambda_{1}(t)\right) \lambda_{0}(t)\right]+2 D^{2}(t) \bar{\varphi}(t) \lambda_{2}(t)=0 .
\end{aligned}
$$

If $\mathcal{R}(x)=\mathcal{R} x$, and $\bar{\phi} \in L^{2}(0, T ; \mathbb{R})$, then (H3) automatically holds. We have $\bar{\Phi}=\bar{\phi} x$ is a closed-loop equilibrium strategy if and only if for $t \in[0, T), D^{2}(t) \lambda_{2}(t) \geq 0$ and

$$
\left[\lambda_{2}(t)-\lambda_{0}^{2}(t)-\frac{1}{2} \mathcal{R} \lambda_{0}(t)\right] B(t)+D^{2}(t) \bar{\phi}(t) \lambda_{2}(t)=0 .
$$

These conclusions coincide with those in $[14,24]$.

Remark 3.4 The condition $D^{2}(t) \lambda_{2}(t) \geq 0$ is a convexity condition for our optimization problem. In the Markovian setting with deterministic $\bar{\phi}$, this conclusion holds by the comparison theorem of ODEs. For $\mathcal{R}(x)=\mathcal{R} x$, we seek the equilibrium strategy with $\bar{\varphi}=0$ to be consistent with the Markovian setting in [3]. When $\mathcal{R}(x)=\mathcal{R}$, the solvability of the relevant backward stochastic Riccati equation (BSRE) system and the existence of $(\bar{\phi}, \bar{\varphi})$ satisfying (H3) are obtained in [22] with the FBSDEs and decoupling techniques, which is different from ours. We leave the solvability of BSRE system when $\mathcal{R}(x)=\mathcal{R} x$ to our future work. 
Remark 3.5 We give some comparisons with the well-known papers [1,3] that treat the Markovian case. First, our Riccati system (3.12) coincides with theirs (see [3, Proposition 4.5]) that are derived from the total variance formula or from [2]. We will show in Subsection 5.2 that, even in the Markovian setting, our approach is different from theirs. Second, the coefficients of the models in $[1,3]$ are constants whereas ours are bounded processes that may be discontinuous (see Theorem 4.2) and/or random, which brings us nontrivial challenges (e.g. the solvability of Riccati system (3.12) due to the rough integrability of $\widetilde{\lambda}_{i}$ ) as well as interesting facts (see e.g. [22]). Third, our assumption (H3) is natural and easy to check in the Markovian setting.

\subsection{Investment/consumption with non-exponential discounting}

Suppose $\left(\Phi_{1}, \Phi_{2}\right)$ are two closed-loop strategies such that for $x \geq 0$, the following SDE admits a unique solution: $s \in[0, T]$,

$$
\begin{aligned}
& d \mathscr{X}(s)=\left[A(s) \mathscr{X}(s)+B(s) \Phi_{2}(s, \mathscr{X}(s))-\Phi_{1}(s, \mathscr{X}(s))\right] d s+D(s) \Phi_{2}(s, \mathscr{X}(s)) d W(s), \\
& \mathscr{X}(0)=x
\end{aligned}
$$

and the following cost functional starting from $(t, \mathscr{X}(t))$ is well-defined,

$$
J(\Phi ; t, \mathscr{X}(t))=\mathbb{E}_{t} \int_{t}^{T} \nu(t, s) U_{1}\left(\Phi_{1}(s, \mathscr{X}(s))\right) d s+\mathbb{E}_{t}\left[\rho(t) U_{2}(\mathscr{X}(T))\right] .
$$

The aim is to maximize $J$ over $\left(\Phi_{1}, \Phi_{2}\right)$. Here $A(\cdot), B(\cdot), D(\cdot)$ are bounded processes, $\nu(t, \cdot)$ is $\mathbb{F}$ adapted, $\rho(t)$ is $\mathcal{F}_{T}$-measurable, and both $\nu, \rho$ are bounded and positive, $U_{1}(x)=U_{2}(x)=x^{\beta}$ with $\beta \in(0,1)$. SDE $(3.13)$ can be interpreted as a wealth equation with $\Phi_{1}(\cdot, \mathscr{X}(\cdot))$ the nonnegative consumption rate and $\Phi_{2}(\cdot, \mathscr{X}(\cdot))$ the amount of wealth invested in risky asset. If $\nu(t, s)=e^{-\alpha(s-t)}$ and $\rho(t)=e^{-\alpha(T-t)}(\alpha>0)$, then (3.14) is a standard power utility maximization problem. If $\nu(t, s)$ and $\rho(t)$ are some other discounting functions, for example, $\nu(t, s)=(1+\alpha(s-t))^{-1}$, the hyperbolic discounting function, then it becomes time-inconsistent. Normally, $\rho(t)=\nu(t, T)$, but can be a different function.

The model (3.13) and the objective (3.14) can be written as (2.1) and (2.2) with

$$
\left\{\begin{array}{l}
b\left(s, x, u_{1}, u_{2}\right)=A(s) x+B(s) u_{2}-u_{1}, \quad \sigma\left(s, x, u_{1}, u_{2}\right)=D(s) u_{2}, \\
g(t, s, \gamma, x, u)=-\nu(t, s) u_{1}^{\beta}, \quad h(t, \gamma, x, y)=-\rho(t) x^{\beta} .
\end{array}\right.
$$

Since $h$ is free of $y$, we do not need to find $\Lambda, \Gamma$ (Remark 3.2). We seek the following ansatz strategy: for $\bar{\phi}_{1} \geq 0$ and $\bar{\phi}_{1}, \bar{\phi}_{2}$ satisfying suitable integrability conditions, any $v_{1} \geq 0, v_{2} \in \mathbb{R}$, define

$$
\bar{\Phi}:=\left(\bar{\Phi}_{1}, \bar{\Phi}_{2}\right)=\left(\bar{\phi}_{1} x, \bar{\phi}_{2} x\right), \quad \Phi^{\prime}:=\left(\left[\bar{\phi}_{1}+v_{1}\right] x,\left[\bar{\phi}_{2}+v_{2}\right] x\right),
$$

and the ansatz solution to BSPDE (3.1) as

$$
\bar{\Xi}(\tau, t, \gamma, y)=Y(\tau, t) y^{\beta}, \quad \bar{\Delta}(\tau, t, \gamma, y)=\tilde{Y}(\tau, t) y^{\beta}
$$

Substituting $(\bar{\Xi}, \bar{\Delta})$ into (3.1), we can obtain the following BSDE:

$$
\begin{aligned}
Y(\tau, t)= & -\rho(\tau)+\int_{t}^{T}\left(\beta Y(\tau, s)\left(A(s)+B(s) \bar{\phi}_{2}(s)-\bar{\phi}_{1}(s)+\frac{1}{2}(\beta-1) D^{2}(s) \bar{\phi}_{2}^{2}(s)\right)\right. \\
& \left.+\beta \widetilde{Y}(\tau, s) D(s) \bar{\phi}_{2}(s)-\nu(\tau, s) \bar{\phi}_{1}^{\beta}(s)\right) d s-\int_{t}^{T} \tilde{Y}(\tau, s) d W(s), t \in[0, T] .
\end{aligned}
$$


(H4) Suppose there exists $\left(\bar{\phi}_{1}, \bar{\phi}_{2}\right)$ such that

(i) $\bar{\phi}_{1}$ is a nonnegative, $\bar{\phi}_{1} \in L_{\mathbb{F}}^{1}\left(\Omega ; L^{1}(0, T ; \mathbb{R})\right), \bar{\phi}_{2} \in L_{\mathbb{F}}^{p}\left(\Omega ; L^{2}(0, T ; \mathbb{R})\right), \forall p>2$.

(ii) $\operatorname{SDE}(2.4)$ admits a unique solution $\overline{\mathscr{X}}(\cdot), \mathscr{X}^{\varepsilon}(\cdot) \in L_{\mathbb{F}}^{p}(\Omega ; C([0, T] ; \mathbb{R})),(p>2)$.

(iii) $\operatorname{BSDE}(3.16)$ admits a solution $(Y(\tau, \cdot), \widetilde{Y}(\tau, \cdot)) \in \mathscr{H}_{1}, \tau \in[0, T]$, such that for almost all $r \in[0, T], \widetilde{Y}(r, r)$ is well-defined.

(iv) $\left(\bar{\phi}_{1}, \bar{\phi}_{2}\right)$ satisfies some limiting conditions in the sense of (A.10).

Denote by $\overline{\mathscr{C}}$ the set of $\Phi^{\prime}$ defined in (3.15) with $\left(\bar{\phi}_{1}, \bar{\phi}_{2}\right)$ satisfying (H4). Clearly, $\overline{\mathscr{C}} \subset \overline{\mathscr{S}}_{a d}$. In this subsection, the equilibrium strategy is defined with $\overline{\mathscr{S}}_{a d}$ replaced by $\overline{\mathscr{C}}$.

Theorem 3.3 Suppose (H4) holds for some $\left(\bar{\phi}_{1}, \bar{\phi}_{2}\right)$. Then $\bar{\Phi}$ in (3.15) is a closed-loop equilibrium strategy if and only if

$$
\begin{aligned}
& Y(t, t) B(t)+(\beta-1) Y(t, t) D^{2}(t) \bar{\phi}_{2}(t)+\tilde{Y}(t, t) D(t)=0, \\
& \nu(t, t)\left[\bar{\phi}_{1}(t)\right]^{\beta-1}+Y(t, t)=0, \quad Y(t, t) \leq 0, \quad t \in[0, T] .
\end{aligned}
$$

Proof. See Section A.3.

Remark 3.6 For $U_{1}(x)=U_{2}(x)=\ln x$, we can easily derive the equilibrium strategy by noting that $\lim _{\beta \rightarrow 0}\left(x^{\beta}-1\right) / \beta=\ln x$ and that, for any fixed $\beta>0$, the equilibrium strategies for $x^{\beta}$ and $\left(x^{\beta}-1\right) / \beta$ are the same. We can give the characterization by setting $\beta=0$ in (3.17), that is,

$$
\begin{aligned}
& Y(t, t) B(t)-Y(t, t) D^{2}(t) \bar{\phi}_{2}(t)+\tilde{Y}(t, t) D(t)=0, \\
& \nu(t, t)\left[\bar{\phi}_{1}(t)\right]^{-1}+Y(t, t)=0, \quad Y(t, t) \leq 0, \quad t \in[0, T],
\end{aligned}
$$

where $(Y, \tilde{Y})$ is the solution to $\operatorname{BSDE}(3.16)$ with $\beta=0$, that is,

$$
Y(\tau, t)=-\rho(\tau)-\int_{t}^{T} \nu(\tau, s) d s-\int_{t}^{T} \tilde{Y}(\tau, s) d W(s), t \in[0, T] .
$$

We can also derive (3.18), (3.19) by the ansatz solution to BSPDE $(3.1)$ as $\bar{\Xi}(\tau, t, y)=Y_{1}(\tau, t) \ln y+$ $Y_{2}(\tau, t), \bar{\Delta}(\tau, t, y)=\widetilde{Y}_{1}(\tau, t) \ln y+\widetilde{Y}_{2}(\tau, t)$. Substituting $(\bar{\Xi}, \bar{\Delta})$ into $(3.1)$, we can get a system of $\operatorname{BSDEs}$ for $\left(Y_{1}, \widetilde{Y}_{1}\right)$ and $\left(Y_{2}, \widetilde{Y}_{2}\right)$, the former satisfies (3.19).

Remark 3.7 We can deduce a number of important facts from our model.

First, in the Markovian setting, $\tilde{Y}=0$. The time inconsistency caused by $\nu, \rho$ does not affect $\bar{\phi}_{2}$, the investment proportion of wealth, for the power and log utility case. It is conjectured in $[6$, Remark 5.5] that such coincidence would disappear in the non-Markovian framework. Here we give a positive answer to this conjecture due to the presence of $\tilde{Y}$.

Second, for log utility model, (3.18) and (3.19) imply that the randomness of equilibrium consumption strategy is only determined by the non-exponential discounting functions but not the other coefficients $A(\cdot), B(\cdot), D(\cdot)$. This interesting feature does not hold for power utility as $\beta \neq 0$, see BSDE (3.16).

Third, in the Markovian setting, if $\nu, \rho$ reduce to exponential discounting functions, from (3.17) and (3.18), the equilibrium strategy $\left(\bar{\phi}_{1}, \bar{\phi}_{2}\right)$ degenerate into the classical optimal strategy, see e.g. [18, Subsection 4.5]. We believe that a similar result also holds in the non-Markovian setting, and leave the details to the interested reader.

Fourth, if $U_{i}(\cdot)=\ln x$, the existence and uniqueness of $(Y, \widetilde{Y})$ for (3.19) is guaranteed. However, if $U_{i}=x^{\beta}$, the well-posedness of (3.16) is yet to be established. In fact, (H4) is standard under the Markovian framework, see e.g. [5, 6, 18], and the related solvability of (3.16) is proved under various cases. In our context, the essential difficulty arises from $\widetilde{Y}$. 


\section{$4 \quad$ Pointwise characterizations with deterministic coefficients}

In this section, we compute the limit in (2.8), using (3.6), and obtain the limit in a delicate way. We demonstrate these ideas from two different viewpoints.

Since the model is Markovian, for a given $\Phi \in \mathscr{S}_{a d}$, instead of the BSPDE (3.1), we introduce the following PDE system: for $0 \leq \tau \leq t \leq T$ and $\gamma, y \in \mathbb{R}^{n}$,

$$
\left\{\begin{array}{l}
\partial_{t} \Lambda(t, y)+(\mathscr{A} \Lambda(t, \cdot))^{\Phi}(y)=0, \\
\partial_{t} \Xi(\tau, t, \gamma, y)+(\mathscr{A} \Xi(\tau, t, \gamma, \cdot))^{\Phi}(y)+g^{\Phi}(\tau, t, \gamma, y)=0, \\
\partial_{t} \Pi(\tau, t, \gamma, y)+(\mathscr{A} \Pi(\tau, t, \gamma, \cdot))^{\Phi}(y)=0, \\
\Lambda(T, y)=y, \Xi(\tau, T, \gamma, y)=h(\tau, \gamma, y, \Lambda(\tau, \gamma)), \\
\Pi(\tau, T, \gamma, y)=\left.\partial_{z} h(\tau, \gamma, y, z)\right|_{z=\Lambda(\tau, \gamma)} .
\end{array}\right.
$$

Note that $\Pi$ is $\mathbb{R}^{1 \times n}$-valued. In contrast with (3.1), we introduce an additional PDE for $\Pi$ which has the following representation under proper conditions,

$$
\left.\mathbb{E}_{t} \partial_{z} h(\tau, \mathscr{X}(\tau), \mathscr{X}(T), z)\right|_{z=\mathbb{E}_{\tau} \mathscr{X}(T)}=\left.\Pi(\tau, t, \gamma, y)\right|_{\gamma=\mathscr{X}(\tau), y=\mathscr{X}(t)} .
$$

Remark 4.1 Given proper functions $k, l$, suppose

$$
h(\tau, \gamma, y, z)=k(\tau, \gamma, y)+l(\tau, \gamma, z), \quad \tau \in[0, T], \gamma, y, z \in \mathbb{R}^{n},
$$

Since $\left.\partial_{z} h(\tau, \gamma, y, z)\right|_{z=\Lambda(\tau, \gamma)}=\left.\partial_{z} l(\tau, \gamma, z)\right|_{z=\Lambda(\tau, \gamma)}$ is independent of $y$, we have a trivial solution to the third PDE of (4.1), that is,

$$
\left.\Pi(\tau, t, \gamma, y) \equiv \partial_{z} l(\tau, \gamma, z)\right|_{z=\Lambda(\tau, \gamma)}, \quad(t, y) \in[0, T] \times \mathbb{R}^{n} .
$$

In particular, if $h$ is independent of $z$, then $\Pi \equiv 0$, and $\Lambda$ is irrelevant (Remark 3.2). Therefore, $\Pi$ is nontrivial only if $y, z$ in $h$ are non-separable.

Example 4.1 Consider the following utility-deviation-risk portfolio selection problem in Wong et al. [27]. Assume $\mathscr{X}$ satisfies the SDE

$$
\left\{\begin{array}{l}
d \mathscr{X}(s)=[A(s) \mathscr{X}(s)+B(s) u(s)] d s+D(s) u(s) d W(s), \quad s \in[0, T], \\
\mathscr{X}(0)=x
\end{array}\right.
$$

where $A, B, D$ are deterministic continuous functions, representing the riskless interest rate, excess stock return, and stock volatility, respectively, $u(s)$ is the amount of money invested in a risky asset and $\mathscr{X}(s)$ the wealth of the investor at time $s$. The objective function is given by

$$
J(u(\cdot) ; t, \mathscr{X}(t)):=\mathbb{E}_{t} U(\mathscr{X}(T))-\gamma \mathbb{E}_{t}\left[\mathcal{D}\left(\mathscr{X}(T)-\mathbb{E}_{t} \mathscr{X}(T)\right)\right],
$$

where $U$ is a continuously differentiable, increasing, strictly concave function and $\mathcal{D}$ a continuously differentiable, nonnegative, convex function with $\mathcal{D}(0)=0$. The utility-deviation-risk portfolio selection problem is to maximize $J$ in (4.5) over $u(\cdot)$ subject to (4.4). [27] discussed the necessary and sufficient optimality conditions and the existence of pre-commitment optimal control for this time-inconsistent problem. To the best knowledge of the authors, the cost functional (4.5) cannot be covered by the existing relevant literature (e.g. [2]). Thanks to the PDE system (4.1) and 
Theorems 4.1 and 4.2 below, we can characterize the closed-loop equilibrium strategy. Specifically, (4.4) and (4.5) can be written as (2.1) and (2.2) with

$$
\left\{\begin{array}{l}
b(s, x, u)=A(s) x+B(s) u, \quad \sigma(s, x, u)=D(s) u, \\
g(t, s, \gamma, x, u)=0, \quad h(t, \gamma, x, y)=-U(x)+\gamma D(x-y) .
\end{array}\right.
$$

Note variables $x, y$ are not separable for general deviation function $\mathcal{D}$ (with one important exception that $\mathcal{D}(x-y)=x^{2}-y^{2}$, variance measure). From Remark 4.1, we need to solve the full PDE system (4.1) for $\Lambda, \Xi, \Pi$ when the equilibrium control $u$ is a closed-loop strategy $u=\Phi(t, x)$. Note also that $\mathrm{Gu}$ et al. [9] investigated a class of utility-deviation-risk portfolio selection problems, but their model can still be cast into a separable structure as that in [2].

Given a closed-loop strategy $\bar{\Phi}$, and solutions $\bar{\Lambda}, \bar{\Xi}, \bar{\Pi}$ to system (4.1), define

$$
\begin{aligned}
H_{d}(t, x, u):= & g(t, t, x, x, u)+\left(\bar{\Pi}(t, t, x, x) \partial_{x} \bar{\Lambda}(t, x)+\left.\partial_{y} \bar{\Xi}(t, t, x, y)\right|_{y=x}\right) b(t, x, u) \\
& +\frac{1}{2} \bar{\Pi}(t, t, x, x) \sigma(t, x, u)^{\top} \partial_{x x} \bar{\Lambda}(t, x) \sigma(t, x, u)+\left.\frac{1}{2} \sigma(t, x, u)^{\top} \partial_{y y} \bar{\Xi}(t, t, x, y)\right|_{y=x} \sigma(t, x, u),
\end{aligned}
$$

with $t \in[0, T], x \in \mathbb{R}^{n}, u \in \mathbb{R}^{m}$. Given state process $\overline{\mathscr{X}}(\cdot)$, the Hamiltonian function $H_{d}(t, \overline{\mathscr{X}}(t), \cdot)$, inspired and formally derived by the pointwise limit of $\mathcal{S}^{\Phi^{\varepsilon}, \bar{\Phi}}(t, \varepsilon)$ in Theorem 3.1 as $\varepsilon \rightarrow 0$, is closely connected with Theorems 4.1 and 4.2 that give two distinctly different pointwise characterizations for closed-loop equilibrium strategies.

\subsection{Characterization I}

The intuitive idea can be easily explained: Given a mapping $f:[0, T] \times[0, T] \rightarrow \mathbb{R}$, consider $\lim _{\varepsilon \rightarrow 0} \int_{t}^{t+\varepsilon} \frac{f(t, s)}{\varepsilon} d s, t \in[0, T)$. If for any $t \in[0, T), s \mapsto f(t, s)$ is continuous, $\sup _{s \in[t, t+\varepsilon]}|f(t, s)|<$ $\infty$, then

$$
\int_{t}^{t+\varepsilon} \frac{|f(t, s)-f(t, t)|}{\varepsilon} d s \leq \sup _{s \in[t, t+\varepsilon]}|f(t, s)-f(t, t)| \rightarrow 0 \text { as } \varepsilon \rightarrow 0,
$$

from which one obtains the limit $f(t, t)$. To adjust this simple idea into our framework, we introduce a subset $\mathscr{D}_{a d}$ of admissible control set $\mathscr{S}_{a d}$ as follows.

Definition 4.1 A map $\Phi:[0, T] \times \mathbb{R}^{n} \rightarrow \mathbb{R}^{n}$ is said to belong to $\mathscr{D}_{\text {ad }}$ if for any $\gamma, x \in \mathbb{R}^{n}, r \in[0, T]$, $s \in[r, T], r \mapsto b^{\Phi}(r, x), \sigma^{\Phi}(r, x), g^{\Phi}(r, s, \gamma, x)$ are right-continuous on $[0, T], x \mapsto g^{\Phi}(r, s, \gamma, x)$ is continuous, and, for some $k \geq 1, K>0$,

$$
\begin{aligned}
& \left|g^{\Phi}(t, s, \gamma, x)\right|+|h(t, \gamma, x, y)| \leq K(1+|\gamma|+|x|+|y|)^{k}, 0 \leq t \leq s \leq T, \gamma, x, y \in \mathbb{R}^{n}, \\
& \left|b^{\Phi}(t, x)\right|+\left|\sigma^{\Phi}(t, x)\right| \leq K(1+|x|), 0 \leq t \leq T, x \in \mathbb{R}^{n}, \\
& \left|b^{\Phi}\left(t, x_{1}\right)-b^{\Phi}\left(t, x_{2}\right)\right|+\left|\sigma^{\Phi}\left(t, x_{1}\right)-\sigma^{\Phi}\left(t, x_{2}\right)\right| \leq K\left|x_{1}-x_{2}\right|, \quad x_{1}, x_{2} \in \mathbb{R}^{n} .
\end{aligned}
$$

A function is said to have polynomial growth if it satisfies an inequality as $g^{\Phi}$ in (4.7). Definition 4.1 is similar to that in He and Jiang [11]. For any $\Phi, \Phi^{\prime} \in \mathscr{D}_{a d}$, and $t \in[0, T)$, we see that $\Phi^{\varepsilon}$ in (2.7) belongs to $\mathscr{D}_{a d}$. Moreover, $\mathscr{D}_{a d} \subset \mathscr{S}_{a d}$. In this subsection, we characterize equilibrium strategy in terms of Definition 2.2 with $\mathscr{S}_{a d}, \overline{\mathscr{S}}_{a d}$ replaced by $\mathscr{D}_{a d}$.

(H5) Suppose for given $t, s \in[0, T], \gamma, x, y \in \mathbb{R}^{n}$, and $\Phi \in \mathscr{D}_{a d},(x, y) \mapsto \partial_{y} h(t, \gamma, x, y),(\gamma, y) \mapsto$ $g^{\Phi}(t, s, \gamma, y),(\gamma, x, y) \mapsto h(t, \gamma, x, y)$ are continuous, $\partial_{y} h$ has polynomial growth. Moreover, there 
exists $\bar{\Phi} \in \mathscr{D}_{a d}$ such that $(4.1)$ admits a solution $(\bar{\Lambda}, \bar{\Xi}, \bar{\Pi})$ satisfying $\bar{\Lambda}(\cdot, \cdot)$ in $C_{n}^{1,2}, \bar{\Xi}(t, \cdot, \gamma, \cdot)$ in $C^{1,2}, \bar{\Pi}(t, \cdot, \gamma, \cdot)$ in $C_{n}^{1,2}$, and $\partial_{x} \bar{\Lambda}, \partial_{x x} \bar{\Lambda}, \partial_{y} \bar{\Xi}, \partial_{y y} \bar{\Xi}, \partial_{y} \bar{\Pi}$ have polynomial growth.

We can now state the second main result of the paper: the pointwise characterization for equilibrium strategy.

Theorem 4.1 Suppose (H5) holds. Then $\bar{\Phi} \in \mathscr{D}_{\text {ad }}$ is a closed-loop equilibrium strategy if and only if, given $H_{d}$ in (4.6),

$$
H_{d}(t, \overline{\mathscr{X}}(t), \bar{\Phi}(t, \overline{\mathscr{X}}(t)))=\min _{\Phi^{\prime} \in \mathscr{D} a d} H_{d}\left(t, \overline{\mathscr{X}}(t), \Phi^{\prime}(t, \overline{\mathscr{X}}(t))\right), \quad \forall t \in[0, T) .
$$

Proof. See Section A.4.

\subsection{Characterization II}

We may drop the right continuity condition of $r \mapsto b^{\Phi}(r, x), \sigma^{\Phi}(r, x), g^{\Phi}(t, r, \gamma, x)$ for any $\Phi \in \mathscr{D}_{a d}$. The intuitive idea again can be easily explained: If $|f(t, s)-f(s, s)| \leq K|t-s|, \forall t, s \in[0, T]$, then

$$
\int_{t}^{t+\varepsilon} \frac{|f(t, s)-f(s, s)|}{\varepsilon} d s \leq \frac{1}{2} K \varepsilon \rightarrow 0 \text { as } \varepsilon \rightarrow 0 .
$$

Using the Lebesgue differentiation theorem, for almost all $t \in[0, T]$, we have

$$
\lim _{\varepsilon \rightarrow 0} \int_{t}^{t+\varepsilon} \frac{f(t, s)}{\varepsilon} d s=\lim _{\varepsilon \rightarrow 0} \int_{t}^{t+\varepsilon} \frac{f(s, s)}{\varepsilon} d s=f(t, t) .
$$

To adjust this simple idea into our framework, we introduce a subset $\mathscr{D}_{a d}^{0}$ of admissible control set $\mathscr{S}_{\text {ad }}$ as follows.

Definition 4.2 A map $\Phi:[0, T] \times \mathbb{R}^{n} \rightarrow \mathbb{R}^{n}$ is said to belong to $\mathscr{D}_{a d}^{0}$ if $b^{\Phi}, \sigma^{\Phi}$ satisfy the two inequalities (linear growth and Lipschitz continuity w.r.t state variable) in (4.7), $h$ has the polynomial growth, and $g^{\Phi}$ satisfies, for some $k \geq 1, K>0$,

$$
\begin{aligned}
& \left|g^{\Phi}\left(t_{1}, r, \gamma_{1}, y_{1}\right)-g^{\Phi}\left(t_{2}, r, \gamma_{2}, y_{2}\right)\right| \\
& \leq K\left[1+\left|\gamma_{1}\right|+\left|\gamma_{2}\right|+\left|y_{1}\right|+\left|y_{2}\right|\right]^{k}\left[\left|y_{1}-y_{2}\right|+\left|t_{1}-t_{2}\right|+\left|\gamma_{1}-\gamma_{2}\right|\right], \\
& \left|g^{\Phi}(t, r, 0,0)\right| \leq K, 0 \leq t \leq r \leq T,\left(t_{i}, \gamma_{i}, y_{i}\right) \in[0, T] \times \mathbb{R}^{n} \times \mathbb{R}^{n}, i=1,2 .
\end{aligned}
$$

A function is said to have Lipschitz continuity with polynomial growth rank if it satisfies two inequalities of the same type as the ones for $g^{\Phi}(\cdot, r, \cdot, \cdot)$ in (4.9). For any $\Phi, \Phi^{\prime} \in \mathscr{D}_{a d}^{0}, t \in[0, T)$, $\Phi^{\varepsilon} \in \mathscr{D}_{a d}^{0}$, and $\mathscr{D}_{a d}^{0} \subset \mathscr{S}_{a d}$. In this subsection, we characterize the equilibrium strategy with $\mathscr{S}_{a d}$, $\overline{\mathscr{S}}_{a d}$ replaced by $\mathscr{D}_{a d}^{0}$.

(H6) Suppose for given $t \in[0, T], \gamma, x, y \in \mathbb{R}^{n},(x, y) \mapsto \partial_{y} h(t, \gamma, x, y)$ are continuous, $(\gamma, x, y) \mapsto$ $\partial_{y} h$ has polynomial growth, and there exists $\bar{\Phi} \in \mathscr{D}_{a d}^{0}$ such that (4.1) admits a solution $(\bar{\Lambda}, \bar{\Xi}, \bar{\Pi})$ satisfying for $t, r \in[0, T], \gamma, y \in \mathbb{R}^{n}, \bar{\Lambda}(\cdot, \cdot)$ in $C_{n}^{1,2}, \bar{\Xi}(t, \cdot, \gamma, \cdot)$ in $C^{1,2}, \bar{\Pi}(t, \cdot, \gamma, \cdot)$ in $C_{n}^{1,2}, \bar{\Pi}$ and $\partial_{y} \bar{\Pi}$ have polynomial growth, and $\partial_{y} \bar{\Xi}(\cdot, r, \cdot, \cdot), \partial_{y y} \bar{\Xi}(\cdot, r, \cdot, \cdot), \partial_{x} \bar{\Lambda}(r, \cdot), \partial_{x x} \bar{\Lambda}(r, \cdot), \bar{\Pi}(\cdot, r, \cdot, y)$ have Lipschitz continuity with polynomial growth.

We next state the third main result of the paper: another pointwsie characterization for equilibrium strategy. 
Theorem 4.2 Suppose (H6) holds. Then $\bar{\Phi} \in \mathscr{D}_{\text {ad }}^{0}$ is a closed-loop equilibrium strategy if and only if, given $H_{d}$ in (4.6),

$$
H_{d}(t, \overline{\mathscr{X}}(t), \bar{\Phi}(t, \overline{\mathscr{X}}(t)))=\min _{\Phi^{\prime} \in \mathscr{D}_{a d}^{0}} H_{d}\left(t, \overline{\mathscr{X}}(t), \Phi^{\prime}(t, \overline{\mathscr{X}}(t))\right), \quad t \in[0, T) \text {. a.e. }
$$

Proof. See Section A.5.

Note the subtle differences of the statements of Theorems 4.1 and 4.2: (4.8) holds for all $t \in[0, T)$ while (4.10) holds for $t \in[0, T)$ almost everywhere.

Remark 4.2 We can deduce some important facts from our model.

First, given $\Phi \in \mathscr{S}_{a d}$, mapping $F:[0, T] \times \mathbb{R}^{n} \rightarrow \mathbb{R}^{n}$, define

$$
\left[\mathcal{A}^{\Phi} F\right](t, x):=\partial_{t} F(t, x)+(\mathscr{A} F(t, \cdot))^{\Phi}(x), \quad(t, x) \in[0, T] \times \mathbb{R}^{n} .
$$

According to (H5) or (H6), for any $(t, x) \in[0, T] \times \mathbb{R}^{n},\left[\mathcal{A}^{\bar{\Phi}} \bar{\Lambda}\right](t, x)=0$, and hence

$$
\left[\mathcal{A}^{\Phi^{\prime}} \bar{\Lambda}\right](t, x)=(\mathscr{A} \bar{\Lambda}(t, \cdot))^{\Phi^{\prime}}(x)-(\mathscr{A} \bar{\Lambda}(t, \cdot))^{\bar{\Phi}}(x) .
$$

A similar conclusion also holds for $\bar{\Xi}$. Recalling (4.6), we have the following result:

$$
\begin{aligned}
& H_{d}\left(t, \overline{\mathscr{X}}(t), \Phi^{\prime}(t, \overline{\mathscr{X}}(t))\right)-H_{d}(t, \overline{\mathscr{X}}(t), \bar{\Phi}(t, \overline{\mathscr{X}}(t))) \\
& =\left.\left(g^{\Phi^{\prime}}(t, t, \overline{\mathscr{X}}(t), x)+\left[\mathcal{A}^{\Phi^{\prime}} \bar{\Xi}(t, \cdot, \overline{\mathscr{X}}(t), \cdot)\right](t, x)+\bar{\Pi}(t, t, \overline{\mathscr{X}}(t), x)\left[\mathcal{A}^{\Phi^{\prime}} \bar{\Lambda}\right](t, x)\right)\right|_{x=\overline{\mathscr{X}}(t)} .
\end{aligned}
$$

The above relation is used in Subsections 5.2 and 5.3.

Second, if all conditions in Definition 4.1 are also satisfied with $\Phi$ being replaced by arbitrary $u \in \mathbb{R}^{m}$, then $u \in \mathscr{D}_{a d}$, and (4.8) can be strengthened as

$$
H_{d}(t, \overline{\mathscr{X}}(t), \bar{\Phi}(t, \overline{\mathscr{X}}(t)))=\min _{u \in \mathbb{R}^{m}} H_{d}(t, \overline{\mathscr{X}}(t), u), \quad \forall t \in[0, T),
$$

in the sense that the infinite dimensional space $\mathscr{D}_{a d}$ is replaced by the finite dimensional space $\mathbb{R}^{m}$. Similar results hold for Definition 4.2 and relation (4.10).

Third, (H5) and (H6) hold true for the two models in Subsections 3.1 and 3.2, if $\bar{\Phi}$ and $\Phi^{\prime}$ are Markovian and take the form of (3.11), (3.15), respectively. For the later model, although $\partial_{y} \bar{\Xi}$, $\partial_{y y} \bar{\Xi}$ do not have Lipschitz continuity with polynomial growth, we have the positive conclusion for $\delta \mathscr{G}_{2}$ which makes the conclusion and approach work as well. Due to the page limit, we omit the details.

Fourth, if we replace $J,\left\{\varepsilon_{n}\right\}$ in $(2.8)$ by $\mathcal{J}, \varepsilon$, respectively, (see e.g. $[2,11]$ ), by the approach developed in Section A.4, the corresponding new equilibrium strategy can be characterized via (4.8), in other words, these two types of equilibrium strategies are equivalent.

Fifth, compared with $[2,11]$, our conclusion is new and more general due to the non-separability of $(x, y)$ in $h(\tau, \gamma, x, y)$ and our approach is distinctly different from theirs and enables us to improve their conclusions, see Subsections 5.2, 5.3.

\section{Comparisons with known models}

In this section we show that our results recover and improve those of the classical optimal control model, the well-known Bjork et al. [2] model, He and Jiang [11] model and Yong [28] model. 


\subsection{Classical optimal control model}

Assume $g, h$ in (2.2) have the forms of $g(t, s, \gamma, x, u) \equiv g(s, x, u), h(t, \gamma, y, z) \equiv h(y)$. This is a time-consistent model. From Remark 4.1, we know $\Pi \equiv 0$. Since the initial time $t$ and state $\gamma$ do not appear in $g, h$, we do not need these two variables. This makes (4.1) much simplified with only $\bar{\Xi}$ that is described as a linear parabolic PDE with given $\bar{\Phi}$ :

$$
\bar{\Xi}(t, y)=h(y)+\int_{t}^{T}\left((\mathscr{A} \bar{\Xi}(s, \cdot))^{\bar{\Phi}}(y)+g^{\bar{\Phi}}(s, y)\right) d s, \quad t \in[0, T], \quad y \in \mathbb{R}^{n} .
$$

$H_{d}(\cdot, \cdot, \cdot)$ reduces to the classical Hamiltonian function, Assumptions (H5) and (H6) become

(H7) $h$ has polynomial growth and there exists $\bar{\Phi} \in \mathscr{D}_{\text {ad }}$ such that the second PDE in (4.1) admits a unique solution $\bar{\Xi}$ such that $\partial_{x} \bar{\Xi}(\cdot, \cdot)$ and $\partial_{x x} \bar{\Xi}(\cdot, \cdot)$ have polynomial growth.

(H8) $h$ has polynomial growth and there exists $\bar{\Phi} \in \mathscr{D}_{a d}^{0}$ such that the second PDE in (4.1) admits a unique solution $\bar{\Xi}$ such that $\partial_{x} \bar{\Xi}(\cdot, \cdot)$ and $\partial_{x x} \bar{\Xi}(\cdot, \cdot)$ have Lipschitz continuity with power growth rank.

If $\bar{\Phi} \in \mathscr{D}_{a d}$ or $\mathscr{D}_{a d}^{0}$ is an equilibrium strategy in the sense that $J, \overline{\mathscr{X}}(t)$ are replaced by $\mathcal{J}, y$, where $y$ is arbitrary element in $\mathbb{R}^{n}$, then $[8$, Theorem 4.1] states, given $(t, y), \bar{\Phi}(\cdot, \overline{\mathscr{X}}(\cdot))$ is optimal on $[t, T]$. In addition, the equilibrium value function $\bar{\Xi}(\cdot, \cdot)$ can be described as

$$
\left\{\begin{array}{l}
\partial_{t} \bar{\Xi}(t, y)+\inf _{u \in \mathbb{R}^{m}}\left\{\frac{1}{2} \sigma(t, y, u)^{\top} \partial_{y y} \bar{\Xi}(t, y) \sigma(t, y, u)+\partial_{y} \bar{\Xi}(t, y) b(t, y, u)+g(t, y, u)\right\}=0, \\
\Xi(T, y)=h(y), \quad t \in[0, T], \quad y \in \mathbb{R}^{n} .
\end{array}\right.
$$

This shows that for time consistent optimal control problems, one may slightly modify Definition 2.2 so that the closed-loop equilibrium strategy is optimal. However, for some specific models, according to [24, Theorem 3.3] and Remark 3.7, Definition 2.2 is indeed enough.

Remark 5.1 Note that the classical HJB equation (5.2) is satisfied by (smooth) value function corresponding to the (open-loop) optimal control, and its derivation relies on the dynamic programming principle, the compact assumption of control region, the delicate properties of classical value functions. In contrast, here (5.2) is satisfied by the equilibrium value function corresponding to the equilibrium strategy, and the aforementioned preparations are not needed. In this sense, our ideas are new and interesting.

\subsection{Bjork et al. [2] model}

Assume $\bar{\Phi}$ is closed-loop equilibrium strategy, $h$ has the separable form (4.3) with $k, l, \partial_{z} l$ being polynomial growth. To keep notational consistency with [2], for each fixed $s \in[0, T], y \in \mathbb{R}^{n}$, we define $f^{s, y}(\cdot, \cdot):=\bar{\Xi}(s, \cdot, y, \cdot)$ and

$$
\begin{aligned}
& f(t, x, s, y):=f^{s, y}(t, x), \quad(l \diamond \bar{\Lambda})(t, x):=l(t, x, \bar{\Lambda}(t, x)), \\
& {\left[\mathcal{H}^{\Phi} \bar{\Lambda}\right](t, x):=\left.\partial_{z} l(t, x, z)\right|_{z=\bar{\Lambda}(t, x)}\left[\mathcal{A}^{\Phi} \bar{\Lambda}\right](t, x) .}
\end{aligned}
$$

Let $s=t, y=\overline{\mathscr{X}}(t)$. Observe that $V(t, \overline{\mathscr{X}}(t))=f(t, \overline{\mathscr{X}}(t), t, \overline{\mathscr{X}}(t))+(l \diamond \bar{\Lambda})(t, \overline{\mathscr{X}}(t))$. Therefore, for $\mathcal{A}^{u}$ defined similarly as in (4.11) with $\Phi$ by $u \in \mathbb{R}^{m}$, we have

$$
\left[\mathcal{A}^{u} V\right](t, \overline{\mathscr{X}}(t))-\left[\mathcal{A}^{u} f(\cdot, \cdot, t, \overline{\mathscr{X}}(t))\right](t, \overline{\mathscr{X}}(t))-\left[\mathcal{A}^{u}(l \diamond \bar{\Lambda})\right](t, \overline{\mathscr{X}}(t)) \equiv 0 .
$$


By Remark 4.1, $\bar{\Pi}(\tau, t, \gamma, y)=\left.\partial_{z} l(\tau, \gamma, z)\right|_{z=\bar{\Lambda}(\tau, \gamma)}$. From (4.12), conclusion (4.13) and (H5), we can rewrite (4.13) as

$$
\left\{\begin{array}{c}
\inf _{u \in \mathbb{R}^{m}}\left\{\left[\mathcal{A}^{u} V\right](t, \overline{\mathscr{X}}(t))+g(t, t, \overline{\mathscr{X}}(t), \overline{\mathscr{X}}(t), u)-\left[\mathcal{A}^{u} f(\cdot, \cdot, t, \overline{\mathscr{X}}(t))\right](t, \overline{\mathscr{X}}(t))\right. \\
\left.+\left[\mathcal{A}^{u} f^{t, \overline{\mathscr{X}}(t)}\right](t, \overline{\mathscr{X}}(t))-\left[\mathcal{A}^{u}(l \diamond \bar{\Lambda})\right](t, \overline{\mathscr{X}}(t))+\left[\mathcal{H}^{u} \bar{\Lambda}\right](t, \overline{\mathscr{X}}(t))\right\}=0
\end{array}\right.
$$

which essentially recovers the main result, the extended HJB system, in [2, Section 6].

Remark 5.2 Compared with [2], our model has the following additional novelties: First, by our developed methodology, in the HJB system we replace classical equilibrium value function (EVF) $V$ by $\bar{\Xi}$ (i.e., the above $\left.f^{s, y}(\cdot, \cdot)\right)$ which has the inherent advantage of the flow property (Remark 3.1). By revealing the interesting relation (5.3), the essential structure of HJB system in [2] is considerably simplified, and some involved assumptions (e.g. the ones on $\mathcal{A}^{u} V, \mathcal{A}^{u} f, \mathcal{A}^{u}(l \diamond \Lambda)$ ) are dropped. Second, our model is more general and can cover utility-deviation-risk model in Example 4.1 while [2] can not. When our setting reduces to [2], we present a new definition of equilibrium strategy (Remark 2.1). Third, in contrast to the Markovian setting in [2], the randomness of coefficients requires us to slightly modify the definition of equilibrium strategy (Remark 2.1), and the derived PDEs system (4.1), a nontrivial extension to that in [2, Definition 6.2], is new due to the presence of $\bar{\Pi}$ (Remark 4.1).

\subsection{He and Jiang [11] model}

Assume $h$ takes the form of (4.3). Given $(\tau, \gamma) \in[0, T] \times \mathbb{R}^{n}$, we next show that, with $\bar{\Phi} \in \mathscr{D}_{\text {ad }}$ satisfying (H5), (4.8) essentially reduces to the analogue conclusion of [11, Theorem 3.3]. From Remark 4.1, $\bar{\Pi}(\tau, t, \gamma, y)=\left.\partial_{z} l(\tau, \gamma, z)\right|_{z=\bar{\Lambda}(\tau, \gamma)}$, therefore, $\partial_{y} \bar{\Pi}(\tau, t, \gamma, y) \equiv 0$. With $\mathcal{A}^{\Phi}$ in (4.11), suppose

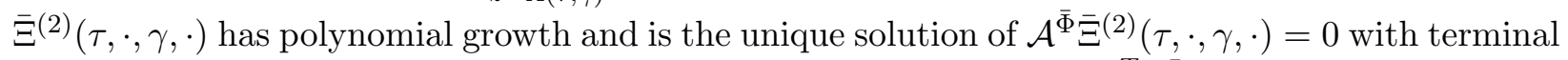
condition $\bar{\Xi}^{(2)}(\tau, T, \gamma, y)=k(\tau, \gamma, y)$. We define $\bar{\Xi}^{(1)}(\tau, t, \gamma, y):=\mathbb{E}_{t} \int_{t}^{T} g^{\bar{\Phi}}(\tau, s, \gamma, \mathscr{M}(s)) d s$, where $\mathscr{M}(\cdot)$ is the solution of $(2.4)$ with $\bar{\Phi}$ and $\mathscr{M}(t)=y$, and the mapping $(t, y) \mapsto \mathcal{G}(t, y ; \tau, s, \gamma)$ as $\mathcal{G}(t, y ; \tau, s, \gamma):=\mathbb{E}_{t} g^{\bar{\Phi}}(\tau, s, \gamma, \mathscr{M}(s)), t \in[\tau, s], y \in \mathbb{R}^{n}$. As in [11], suppose that $\mathcal{G}$ is the solution of $\left[\mathcal{A}^{\bar{\Phi}} \mathcal{G}(\cdot, \cdot ; \tau, s, \gamma)\right]=0$ with $\mathcal{G}(s, y ; \tau, s, \gamma)=g^{\Phi}(\tau, s, \gamma, y)$ such that $\partial_{t} \mathcal{G}, \partial_{y} \mathcal{G}, \partial_{y y} \mathcal{G}$ have polynomial growth. Then $\Xi^{(1)}(\tau, t, \gamma, y)=\int_{t}^{T} \mathcal{G}(t, y ; \tau, s, \gamma) d s$ and $\partial_{y} \bar{\Xi}^{(1)}, \partial_{y y} \bar{\Xi}^{(1)}$ have polynomial growth too. In addition, for any $\Phi \in \mathscr{D}_{a d}$,

$$
\left[\mathcal{A}^{\Phi} \bar{\Xi}^{(1)}(\tau, \cdot, \gamma, \cdot)\right](t, y)=\int_{t}^{T}\left[\mathcal{A}^{\Phi} \mathcal{G}(\cdot, \cdot ; \tau, s, \gamma)\right](t, y) d s-g^{\bar{\Phi}}(\tau, t, \gamma, y)
$$

In particular, by taking $\Phi=\bar{\Phi}$, we arrive at $\left[\mathcal{A}^{\bar{\Phi}} \bar{\Xi}^{(1)}(\tau, \cdot, \gamma, \cdot)\right]+g^{\bar{\Phi}}(\tau, \cdot, \gamma, \cdot)=0$ with $\bar{\Xi}^{(1)}(\tau, T, \gamma, y)=$ 0 . Simple calculation shows that for $\tau \in[0, T], \gamma \in \mathbb{R}^{m}, j=1,2$, the following defined $\Xi(\tau, \cdot, \gamma, \cdot)$ satisfies the second PDE in (4.1) associated with $\bar{\Phi}$,

$$
\bar{\Xi}(\tau, \cdot, \gamma, \cdot):=\bar{\Xi}^{(1)}(\tau, \cdot, \gamma, \cdot)+\bar{\Xi}^{(2)}(\tau, \cdot, \gamma, \cdot)+l(\tau, \gamma, \bar{\Lambda}(\tau, \gamma))
$$

The polynomial growth of $\bar{\Lambda}, l, \bar{\Xi}^{(1)}, \bar{\Xi}^{(2)}$ implies that of $\bar{\Xi}$. Recalling $(4.12)$, we obtain that $\bar{\Phi}(\cdot, \cdot)$ is the equilibrium strategy if and only if

$$
\begin{aligned}
& \min _{\Phi^{\prime} \in \mathscr{D}_{a d}}\left\{g^{\Phi^{\prime}}(t, t, \gamma, x)-g^{\bar{\Phi}}(t, t, \gamma, x)+\int_{t}^{T}\left[\mathcal{A}^{\Phi^{\prime}} \mathcal{G}(\cdot, \cdot ; t, s, \gamma)\right](t, x) d s\right.
\end{aligned}
$$

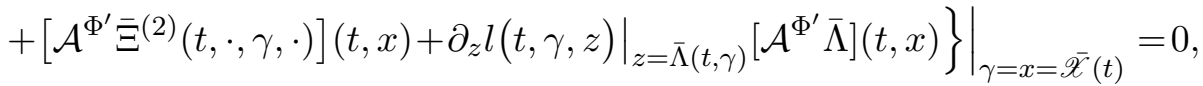


which is essentially consistent with the main result in [11, Theorem 3.3].

Remark 5.3 We highlight some key differences and contributions of our paper compared with [11]. First, our framework is more general to cover Example 4.1 while [11] can not. Second, our developed approach in dealing with the intertemporal cost functional is different from [11] that follows [5]. The additional assumptions on $\mathcal{G}, \partial_{t} \mathcal{G}, \partial_{y} \mathcal{G}, \partial_{y y} \mathcal{G}$ are not needed in our case. Third, by Theorem 4.2 we can drop the right continuity of coefficients imposed in [11]. For example, as to mean-variance problem or investment/consumption problem, this implies that the interest rate, the excess stock return, the stock volatility do not have to be continuous. Fourth, we replace the abstract notion of "support of distributions" in [11] by the value of equilibrium state process to bypass the issue in [11, Footnote 6]. As a trade-off, we need the continuity assumption of $g^{\Phi}, h$ in (H5) which is fulfilled for most well-known models.

\subsection{Yong [28] model}

It is a special form of the cost functional (2.2) with $g(t, s, X(s), u(s))$ and $h(t, X(T))$. For $\tau, t \in$ $[0, T], x \in \mathbb{R}^{n}, u \in \mathbb{R}^{m}, p \in \mathbb{R}^{n}, P \in \mathbb{R}^{n \times n}$, define the Hamiltonian function $\mathbb{H}$, same as [28], by $\mathbb{H}(\tau, t, x, u, p, P):=p^{\top} b(t, x, u)+\frac{1}{2} \sigma(t, x, u)^{\top} P \sigma(t, x, u)+g(\tau, t, x, u)$. Suppose there exists a well-defined mapping $\psi:[0, T]^{2} \times \mathbb{R}^{n} \times \mathbb{R}^{n} \times \mathbb{R}^{n \times n}$ such that

$$
\mathbb{H}(\tau, t, x, \psi(\tau, t, x, p, P), p, P)=\inf _{u \in \mathbb{R}^{m}} \mathbb{H}(\tau, t, x, u, p, P)>-\infty,
$$

and the following PDE admits a classical solution $\Sigma$

$$
\left\{\begin{array}{l}
\partial_{t} \bar{\Sigma}(\tau, t, y)+(\mathscr{A} \bar{\Sigma}(\tau, t, \cdot))^{\bar{\Phi}}(y)+g^{\bar{\Phi}}(\tau, t, y)=0, \quad t \in[\tau, T], y \in \mathbb{R}^{n} \\
\bar{\Sigma}(\tau, T, y)=h(\tau, y), \quad t \in[\tau, T], y \in \mathbb{R}^{n}, \\
\bar{\Phi}(t, y):=\psi\left(t, t, y, \bar{\Sigma}_{y}(t, t, y), \bar{\Sigma}_{y y}(t, t, y)\right), \quad t \in[0, T], y \in \mathbb{R}^{n} .
\end{array}\right.
$$

Using this $\bar{\Phi}$, we have $\partial_{t} \bar{\Xi}(\tau, \cdot, \cdot)+(\mathscr{A} \bar{\Xi}(\tau, \cdot, \cdot))^{\bar{\Phi}}(\cdot)+g^{\bar{\Phi}}(\tau, \cdot, \cdot)=0$ with $\bar{\Xi}(\tau, t, y)=h(\tau, y)$. By the uniqueness of PDEs, for any $\tau, \bar{\Sigma}(\tau, \cdot, \cdot)=\Xi(\tau, \cdot, \cdot)$. In terms of (4.6), (5.5) and $\bar{\Phi}$ in (5.6), we have $H_{d}(t, x, \bar{\Phi}(t, x))=\inf _{u \in \mathbb{R}^{m}} H_{d}(t, x, u)$, with $x \in \mathbb{R}^{n}$. Thanks to (4.13) and (H3), it follows from Theorem 4.1 that $\bar{\Phi}$ is a closed-loop equilibrium strategy. One can also verify this by Theorem 4.2 with minor modifications. In summary, the PDE in (5.6), the so-called equilibrium HJB equation in [28], coincides with our PDE for $\bar{\Xi}$. [28] also directly uses $\bar{\Sigma}$ which equals to our auxiliary equilibrium value function $\bar{\Xi}$.

There are some essential differences between [28] and this paper. First, the ways of defining the closed-loop equilibrium strategy are different. In [28] some closed-loop strategy for any partition is first constructed by sticking together the obtained Nash equilibrium on each small interval and then the equilibrium strategy is defined as the limit in a subtle way. In contrast, our definition is simpler and the limit techniques focus on the difference of cost functional, rather than the equilibrium strategy. Second, the approaches are different. In [28], for any given partition, the multi-person non-cooperative differential games ideas are first used to obtain the Nash equilibrium by the classical HJB equation and FBSDEs. Then the equilibrium HJB equation is formally obtained by passing the size of partition to zero, and finally a rigorous verification is provided under special cases. These procedures are completely different from ours.

\section{Conclusions}

In this paper we propose a general framework for time-inconsistent optimal control problems with random coefficients. We develop a new approach to characterizing the closed-loop equilibrium 
strategies in both integral and pointwise forms and apply the results to solve the mean-variance problem with state-dependent risk aversion and the investment/consumption problem with nonexponential discounting. We compare, recover and improve the results of some well-known models in the literature and highlight the key advantages of our approach and distinct features of our model. There remain many open questions for time-inconsistent models, including the well-posedness of the BSPDE (3.1) with a given admissible strategy and that of the BSDE (3.16). We leave these questions and others for future research.

Acknowledgments. The authors are very grateful to two anonymous reviewers whose constructive comments and suggestions have helped to improve the paper of the previous version.

\section{A Proofs of theorems}

\section{A.1 Proof of Theorem 3.1}

Since $(\bar{\Lambda}, \bar{\Gamma})$ satisfies (3.1) with $\bar{\Phi}$, by Itô-Kunita formula to $\bar{\Lambda}(r, \overline{\mathscr{X}}(r))$ on $[t, T]$, and integrability assumptions (2.5), (3.4), we have $\mathbb{E}_{t} \overline{\mathscr{X}}(T)=\bar{\Lambda}(t, \overline{\mathscr{X}}(t))$. Because $(\bar{\Xi}, \bar{\Delta})$ satisfies (3.1) with $\bar{\Phi}$, by Itô-Kunita formula to $\bar{\Xi}\left(t, r, \overline{\mathscr{X}}(t), \mathscr{X}^{\varepsilon}(r)\right)$ on $[t+\varepsilon, T]$, and relevant integrability assumptions in $(2.5),(3.4),(3.5)$,

$$
\begin{aligned}
& \bar{\Xi}\left(t, t+\varepsilon, \overline{\mathscr{X}}(t), \mathscr{X}^{\varepsilon}(t+\varepsilon)\right) \\
& =\mathbb{E}_{t+\varepsilon}\left[h\left(t, \overline{\mathscr{X}}(t), \mathscr{X}^{\varepsilon}(T), \mathbb{E}_{t} \overline{\mathscr{X}}(T)\right)+\int_{t+\varepsilon}^{T} g^{\bar{\Phi}}\left(t, s, \overline{\mathscr{X}}(t), \mathscr{X}^{\varepsilon}(s)\right) d s\right] .
\end{aligned}
$$

Similarly, one obtains (A.1) with $\mathscr{X}^{\varepsilon}(t+\varepsilon)$ replaced by $\overline{\mathscr{X}}(t+\varepsilon)$.

Given $\Phi^{\varepsilon} \in \mathscr{S}_{a d}$, and the associated $\Theta_{0}$ in (3.7),

$$
J\left(\Phi^{\varepsilon} ; t, \overline{\mathscr{X}}(t)\right)=\mathbb{E}_{t}\left[\int_{t}^{t+\varepsilon} g^{\Phi^{\prime}}\left(t, s, \overline{\mathscr{X}}(t), \mathscr{X}^{\varepsilon}(s)\right) d s+\Theta_{0}\left(t, t+\varepsilon, \overline{\mathscr{X}}(t), \mathscr{X}^{\varepsilon}(t+\varepsilon)\right)\right] .
$$

Observe that $\Phi^{\varepsilon}(\cdot, x)=\bar{\Phi}(\cdot, x), \mathscr{X}^{\varepsilon}(\cdot)=\overline{\mathscr{X}}^{t+\varepsilon, \mathscr{X}^{\varepsilon}(t+\varepsilon)}(\cdot)$ on $[t+\varepsilon, T]$, and (A.1), we have

$$
\begin{aligned}
& \Theta_{0}\left(t, t+\varepsilon, \overline{\mathscr{X}}(t), \mathscr{X}^{\varepsilon}(t+\varepsilon)\right) \\
& =\mathbb{E}_{t+\varepsilon} \int_{t+\varepsilon}^{T} g^{\bar{\Phi}}\left(t, s, \overline{\mathscr{X}}(t), \mathscr{X}^{\varepsilon}(s)\right) d s+\mathbb{E}_{t+\varepsilon}[H(t, \varepsilon)] \mathbb{E}_{t}\left[\mathscr{X}^{\varepsilon}(T)-\mathscr{X}(T)\right] \\
& \quad+\mathbb{E}_{t+\varepsilon} h\left(t, \overline{\mathscr{X}}(t), \mathscr{X}^{\varepsilon}(T), \mathbb{E}_{t} \overline{\mathscr{X}}(T)\right) \\
& =\mathbb{E}_{t+\varepsilon}[H(t, \varepsilon)] \mathbb{E}_{t}\left[\mathscr{X}^{\varepsilon}(T)-\overline{\mathscr{X}}(T)\right]+\bar{\Xi}\left(t, t+\varepsilon, \overline{\mathscr{X}}(t), \mathscr{X}^{\varepsilon}(t+\varepsilon)\right),
\end{aligned}
$$

where $H(t, \varepsilon)$ is defined in (3.3), and all the terms are well-defined due to the integrability in (2.5) and (3.5). Similar as the above, we have

$$
J(\bar{\Phi} ; t, \overline{\mathscr{X}}(t))=\mathbb{E}_{t}\left[\int_{t}^{t+\varepsilon} g^{\bar{\Phi}}(t, s, \overline{\mathscr{X}}(t), \overline{\mathscr{X}}(s)) d s+\bar{\Xi}(t, t+\varepsilon, \overline{\mathscr{X}}(t), \overline{\mathscr{X}}(t+\varepsilon))\right] .
$$

As a result, we have

$$
\begin{aligned}
& J\left(\Phi^{\varepsilon} ; t, \overline{\mathscr{X}}(t)\right)-J(\bar{\Phi} ; t, \overline{\mathscr{X}}(t)) \\
& =\mathbb{E}_{t}\left[\int_{t}^{t+\varepsilon}\left(g^{\Phi^{\prime}}\left(t, s, \overline{\mathscr{X}}(t), \mathscr{X}^{\varepsilon}(s)\right)-g^{\bar{\Phi}}(t, s, \overline{\mathscr{X}}(t), \overline{\mathscr{X}}(s))\right) d s\right] \\
& \quad+\mathbb{E}_{t}\left[H(t, \varepsilon) \mathbb{E}_{t}\left[\mathscr{X}^{\varepsilon}(T)-\overline{\mathscr{X}}(T)\right]\right] \\
& \quad+\mathbb{E}_{t}\left[\bar{\Xi}\left(t, t+\varepsilon, \overline{\mathscr{X}}(t), \mathscr{X}^{\varepsilon}(t+\varepsilon)\right)-\bar{\Xi}(t, t+\varepsilon, \overline{\mathscr{X}}(t), \overline{\mathscr{X}}(t+\varepsilon))\right] .
\end{aligned}
$$


By BSPDEs (3.1), (H2), the notations in (3.2), (3.3), and the well-known Itô-Kunita formula, we derive $\mathbb{E}_{t}[\bar{\Lambda}(t, \overline{\mathscr{X}}(t))-\bar{\Lambda}(t+\varepsilon, \overline{\mathscr{X}}(t+\varepsilon))]=0$ and

$$
\mathbb{E}_{t}\left[\bar{\Lambda}\left(t+\varepsilon, X^{\varepsilon}(t+\varepsilon)\right)-\bar{\Lambda}\left(t, \mathscr{X}^{\varepsilon}(t)\right)\right]=\mathbb{E}_{t} \int_{t}^{t+\varepsilon} \delta \mathscr{G}_{1}\left(r, \mathscr{X}^{\varepsilon}(r)\right) d r
$$

Here the stochastic integral disappear due to (3.4). Recall that $\overline{\mathscr{X}}(t)=\mathscr{X}^{\varepsilon}(t)$, we thus have

$$
\mathbb{E}_{t}\left[\mathscr{X}^{\varepsilon}(T)-\overline{\mathscr{X}}(T)\right]=\mathbb{E}_{t} \int_{t}^{t+\varepsilon} \delta \mathscr{G}_{1}\left(r, \mathscr{X}^{\varepsilon}(r)\right) d r .
$$

As for $\bar{\Xi}$, using BSPDEs (3.1), (H2), the notations in (3.2), (3.3), and the Itô-Kunita formula, we derive that

$$
\begin{aligned}
& \mathbb{E}_{t}\left[\bar{\Xi}\left(t, t+\varepsilon, \overline{\mathscr{X}}(t), \mathscr{X}^{\varepsilon}(t+\varepsilon)\right)-\bar{\Xi}(t, t, \overline{\mathscr{X}}(t), \overline{\mathscr{X}}(t))\right] \\
& =\mathbb{E}_{t}\left[\int_{t}^{t+\varepsilon} \delta \mathscr{G}_{2}\left(t, r, \overline{\mathscr{X}}(t), \mathscr{X}^{\varepsilon}(r)\right) d r-\int_{t}^{t+\varepsilon} g^{\bar{\Phi}}\left(t, r, \overline{\mathscr{X}}(t), \mathscr{X}^{\varepsilon}(r)\right) d r\right], \\
& \mathbb{E}_{t}[\bar{\Xi}(t, t+\varepsilon, \overline{\mathscr{X}}(t), \overline{\mathscr{X}}(t+\varepsilon))-\bar{\Xi}(t, t, \overline{\mathscr{X}}(t), \overline{\mathscr{X}}(t))] \\
& =-\mathbb{E}_{t} \int_{t}^{t+\varepsilon} g^{\bar{\Phi}}(t, r, \bar{X}(t), \overline{\mathscr{X}}(r)) d r .
\end{aligned}
$$

Then conclusion (3.6) follows from (A.3), (A.4), Definition 2.2 and

$$
\begin{aligned}
& \mathbb{E}_{t}\left[\Xi\left(t, t+\varepsilon, \overline{\mathscr{X}}(t), \mathscr{X}^{\varepsilon}(t+\varepsilon)\right)-\bar{\Xi}(t, t+\varepsilon, \overline{\mathscr{X}}(t), \overline{\mathscr{X}}(t+\varepsilon))\right] \\
& =\mathbb{E}_{t}\left[\int_{t}^{t+\varepsilon}\left(\delta \overline{\mathscr{G}}_{2}\left(t, r, \overline{\mathscr{X}}(t), \mathscr{X}^{\varepsilon}(r)\right)-g^{\bar{\Phi}}\left(t, r, \overline{\mathscr{X}}(t), \mathscr{X}^{\varepsilon}(r)\right)+g^{\bar{\Phi}}(t, r, \overline{\mathscr{X}}(t), \overline{\mathscr{X}}(r))\right) d r\right] .
\end{aligned}
$$

The other conclusion is easy to see.

\section{A.2 Proof of Theorem 3.2}

By the definition of $\left(\bar{\Phi}, \Phi^{\prime}\right)$ in $(3.11)$, we see that all the conditions in (H2) are satisfied under (H3). For notational simplicity, given mapping $f, g$, denote by $\mathscr{K}_{f, g}:=B f+D g$. Note that $\delta \mathscr{G}_{1}\left(r, \mathscr{X}^{\varepsilon}(r)\right)=\mathscr{K}_{\lambda_{0}, \tilde{\lambda}_{0}}(r) v$, and

$$
\begin{aligned}
\delta \mathscr{G}_{2}\left(r, \mathscr{X}^{\varepsilon}(r)\right)= & {\left[2 \mathscr{X}^{\varepsilon}(r) \mathscr{K}_{\lambda_{2}, \widetilde{\lambda}_{2}}(r)+\mathscr{K}_{\lambda_{3}, \widetilde{\lambda}_{3}}(r)-\mathcal{R}(\overline{\mathscr{X}}(t)) \mathscr{K}_{\lambda_{0}, \widetilde{\lambda}_{0}}(r)\right] v } \\
& +2 \lambda_{2}(r) D^{2}(r)\left[2 \bar{\phi}(r) \mathscr{X}^{\varepsilon}(r) v+v^{2}+2 \bar{\varphi}(r) v\right] .
\end{aligned}
$$

As a result, (3.6) can be rewritten as

$$
\begin{aligned}
& J\left(\Phi^{\varepsilon} ; t, \mathscr{X}^{\varepsilon}(t)\right)-J(\bar{\Phi} ; t, \overline{\mathscr{X}}(t)) \\
& =\mathbb{E}_{t} \int_{t}^{t+\varepsilon}\left(H(t, \varepsilon) \mathscr{K}_{\lambda_{0}, \widetilde{\lambda}_{0}}(r)+2 \mathscr{X}^{\varepsilon}(r) \mathscr{K}_{\lambda_{2}, \widetilde{\lambda}_{2}}(r)+\mathscr{K}_{\lambda_{3}, \widetilde{\lambda}_{3}}(r)-\mathcal{R}(\overline{\mathscr{X}}(t)) \mathscr{K}_{\lambda_{0}, \widetilde{\lambda}_{0}}(r)\right) v d r \\
& \quad+\mathbb{E}_{t} \int_{t}^{t+\varepsilon} \lambda_{2}(r) D^{2}(r)\left(2 \bar{\phi}(r) \mathscr{X}^{\varepsilon}(r) v+v^{2}+2 \bar{\varphi}(r) v\right) d r .
\end{aligned}
$$


To show the limiting condition for $\bar{\Phi}$, for any sequence $\varepsilon_{n}$ satisfying $\lim _{n \rightarrow \infty} \varepsilon_{n}=0$, we assume that $\overline{\mathscr{X}}^{\varepsilon_{n}}(\cdot), \overline{\mathscr{X}}(\cdot)$ satisfy: for any $t \in[0, T), t+\varepsilon_{n} \leq T, p>2$,

$$
\left\{\begin{array}{l}
\mathbb{E}_{t} \sup _{s \in\left[t, t+\varepsilon_{n}\right]}\left|\mathscr{X}^{\varepsilon_{n}}(s)-\overline{\mathscr{X}}(s)\right|^{p} \leq K \varepsilon_{n}^{\frac{p}{2}} \\
\mathbb{E}_{t} \sup _{s \in\left[t+\varepsilon_{n}, T\right]}\left|\mathscr{X}^{\varepsilon_{n}}(s)-\overline{\mathscr{X}}(s)\right|^{p} \leq K \mathbb{E}_{\tau}\left|\mathscr{X}^{\varepsilon_{n}}\left(t+\varepsilon_{n}\right)-\overline{\mathscr{X}}\left(t+\varepsilon_{n}\right)\right|^{p} .
\end{array}\right.
$$

Moreover, for $(f, \widetilde{f}):=\left(\lambda_{0}, \widetilde{\lambda}_{0}\right),\left(\lambda_{2} \overline{\mathscr{X}}, \widetilde{\lambda}_{2} \overline{\mathscr{X}}\right),\left(\lambda_{3}, \widetilde{\lambda}_{3}\right)$, suppose

$$
\left\{\begin{array}{l}
\lim _{\varepsilon_{n} \rightarrow 0} \frac{1}{\varepsilon_{n}} \mathbb{E}_{t}\left[\int_{t}^{t+\varepsilon_{n}} \mathscr{K}_{f, \tilde{f}}(r) d r\right]=\mathscr{K}_{f, \tilde{f}}(t), \\
\lim _{\varepsilon_{n} \rightarrow 0} \frac{1}{\varepsilon_{n}} \mathbb{E}_{t}\left[\int_{t}^{t+\varepsilon_{n}} \lambda_{2}(r)\left(2 D^{2}(r)[\bar{\phi}(r) \overline{\mathscr{X}}(r)+\bar{\varphi}(r)] v+D^{2}(r) v^{2}\right) d r\right] \\
=\lambda_{2}(t)\left(2 D^{2}(t)[\bar{\phi}(t) \overline{\mathscr{X}}(t)+\bar{\varphi}(t)] v+D^{2}(t) v^{2}\right) .
\end{array}\right.
$$

As a result of (A.5), we have

$$
\lim _{\varepsilon_{n} \rightarrow 0} H\left(t, \varepsilon_{n}\right)=-2 \mathbb{E}_{t} \overline{\mathscr{X}}(T)=-2 \bar{\Lambda}(t, \overline{\mathscr{X}}(t))=-2 \lambda_{0}(t) \overline{\mathscr{X}}(t)-2 \lambda_{1}(t)
$$

In addition,

$$
\mathbb{E}_{t} \int_{t}^{t+\varepsilon_{n}}\left|\mathscr{K}_{\lambda_{2}, \widetilde{\lambda}_{2}}(r)\right|\left|\mathscr{X}^{\varepsilon_{n}}(r)-\overline{\mathscr{X}}(r)\right| d r \leq K \varepsilon_{n}\left[\mathbb{E}_{t} \int_{t}^{t+\varepsilon_{n}}\left|\mathscr{K}_{\lambda_{2}, \widetilde{\lambda}_{2}}(r)\right|^{2} d r\right]^{\frac{1}{2}} .
$$

Similarly,

$$
\lim _{\varepsilon_{n} \rightarrow 0} \frac{1}{\varepsilon_{n}} \mathbb{E}_{t} \int_{t}^{t+\varepsilon_{n}} \lambda_{2}(r) D^{2}(r) \bar{\phi}(r)\left[\mathscr{X}^{\varepsilon_{n}}(r)-\overline{\mathscr{X}}(r)\right] d r=0 .
$$

We discuss the two cases of $\mathcal{R}(\cdot)$. If $\mathcal{R}(\cdot) \equiv \mathcal{R}$ is a constant, then from (A.7), (A.8), (A.9), (A.6), we have

$$
\begin{aligned}
& \lim _{\varepsilon_{n} \rightarrow 0} \frac{J\left(\Phi^{\varepsilon_{n}} ; t, \overline{\mathscr{X}}(t)\right)-J(\bar{\Phi} ; t, \overline{\mathscr{X}}(t))}{\varepsilon_{n}} \\
& =\left[2 \overline{\mathscr{X}}(t)\left\{\mathscr{K}_{\lambda_{2}, \widetilde{\lambda}_{2}}(t)-\lambda_{0}(t) \mathscr{K}_{\lambda_{0}, \widetilde{\lambda}_{0}}(t)+D^{2}(t) \bar{\phi}(t) \lambda_{2}(t)\right\}+\mathscr{K}_{\lambda_{3}, \widetilde{\lambda}_{3}}(t)\right. \\
& \left.\quad-\mathcal{R} \mathscr{K}_{\lambda_{0}, \widetilde{\lambda}_{0}}(t)-2 \lambda_{1}(t) \mathscr{K}_{\lambda_{0}, \widetilde{\lambda}_{0}}(t)+2 D^{2}(t) \bar{\varphi}(t) \lambda_{2}(t)\right] v+D^{2}(t) \lambda_{2}(t) v^{2} .
\end{aligned}
$$

The first part of Theorem 3.2 is easy to see.

If $\mathcal{R}(\gamma)=\mathcal{R} \gamma$ with constant $\mathcal{R}>0, \bar{\varphi}=0$. Then $\left(\lambda_{1}, \widetilde{\lambda}_{1}, \lambda_{3}, \widetilde{\lambda}_{3}\right)=(0,0,0,0)$,

$$
\begin{aligned}
& \lim _{\varepsilon_{n} \rightarrow 0} \frac{J\left(\Phi^{\varepsilon_{n}} ; t, \overline{\mathscr{X}}(t)\right)-J(\bar{\Phi} ; t, \overline{\mathscr{X}}(t))}{\varepsilon_{n}} \\
& =2 \overline{\mathscr{X}}(t)\left\{\mathscr{K}_{\lambda_{2}, \widetilde{\lambda}_{2}}(t)-\lambda_{0}(t) \mathscr{K}_{\lambda_{0}, \tilde{\lambda}_{0}}(t)+D^{2}(t) \bar{\phi}(t) \lambda_{2}(t)-\frac{1}{2} \mathcal{R} \mathscr{K}_{\lambda_{0}, \widetilde{\lambda}_{0}}(t)\right\} v+D^{2}(t) \lambda_{2}(t) v^{2} .
\end{aligned}
$$

The second part of Theorem 3.2 is easy to see. 


\section{A.3 Proof of Theorem 3.3}

After some direct calculations, we know that

$$
\bar{\Xi}(\tau, t, y)=Y(\tau, t) y^{\beta}, \quad \bar{\Delta}(\tau, t, y)=\tilde{Y}(\tau, t) y^{\beta},
$$

satisfies the second BSPDE in (3.1) corresponding to $\left(\bar{\phi}_{1}, \bar{\phi}_{2}\right)$. In addition, under (H6), (H2) holds naturally. It then follows from Theorem 3.1 that

$$
\begin{aligned}
& J\left(\Phi^{\varepsilon_{n}} ; t, \overline{\mathscr{X}}(t)\right)-J(\bar{\Phi} ; t, \overline{\mathscr{X}}(t)) \\
& =\mathbb{E}_{t} \int_{t}^{t+\varepsilon_{n}}\left[\mathscr{X}^{\varepsilon_{n}}(r)\right]^{\beta}\left\{\mathscr{H}_{1}\left(t, r, v_{1}\right)+\mathscr{H}_{2}(t, r) v_{2}+\mathscr{H}_{3}(t, r) v_{2}^{2}\right\} d r,
\end{aligned}
$$

where

$$
\left\{\begin{array}{l}
\mathscr{H}_{1}\left(t, r, v_{1}\right):=-\nu(t, r)\left[\bar{\phi}_{1}(r)+v_{1}\right]^{\beta}+\nu(t, r)\left[\bar{\phi}_{1}(r)\right]^{\beta}-\beta Y(t, r) v_{1}, \\
\mathscr{H}_{2}(t, r):=\beta Y(t, r) B(r)+\beta(\beta-1) Y(t, r) D^{2}(r) \bar{\phi}_{2}(r)+\beta \widetilde{Y}(t, r) D(r), \\
\mathscr{H}_{3}(t, r):=\frac{\beta(\beta-1)}{2} Y(t, r) D^{2}(r)
\end{array}\right.
$$

To present the limiting conditions of $\left(\bar{\phi}_{1}, \bar{\phi}_{2}\right)$, we suppose that

$$
\left\{\begin{array}{l}
\lim _{\varepsilon_{n} \rightarrow 0} \mathbb{E}_{t} \int_{t}^{t+\varepsilon_{n}} \frac{\mathscr{H}_{1}\left(t, r, v_{1}\right)\left[\mathscr{X}^{\varepsilon_{n}}(r)\right]^{\beta}}{\varepsilon_{n}} d r=\mathscr{H}_{1}\left(t, t, v_{1}\right)[\overline{\mathscr{X}}(t)]^{\beta}, \\
\lim _{\varepsilon_{n} \rightarrow 0} \mathbb{E}_{t} \int_{t}^{t+\varepsilon_{n}} \frac{\mathscr{H}_{i}(t, r)\left[\mathscr{X}^{\varepsilon_{n}}(r)\right]^{\beta}}{\varepsilon_{n}} d r=\mathscr{H}_{i}(t, t)[\overline{\mathscr{X}}(t)]^{\beta}, \quad i=2,3 .
\end{array}\right.
$$

Recall that $\mathscr{X}^{\varepsilon_{n}}(\cdot)$ is solution of $(2.4)$ associated with $\Phi^{\varepsilon_{n}}$. As a result,

$$
\begin{aligned}
& \lim _{\varepsilon_{n} \rightarrow 0} \frac{J\left(\Phi^{\varepsilon_{n}} ; t, \overline{\mathscr{X}}(t)\right)-J(\bar{\Phi} ; t, \overline{\mathscr{X}}(t))}{\varepsilon_{n}} \\
& =[\overline{\mathscr{X}}(t)]^{\beta}\left\{\mathscr{H}_{1}\left(t, t, v_{1}\right)+\mathscr{H}_{2}(t, t) v_{2}+\mathscr{H}_{3}(t, t) v_{2}^{2}\right\} .
\end{aligned}
$$

Therefore, $\left(\bar{\phi}_{1}, \bar{\phi}_{2}\right)$ is equilibrium strategy if and only if for any $v_{1} \geq 0, v_{2} \in \mathbb{R}$,

$$
\mathscr{H}_{1}\left(t, t, v_{1}\right)+\mathscr{H}_{2}(t, t) v_{2}+\mathscr{H}_{3}(t, t) v_{2}^{2} \geq 0 \text {. }
$$

By the definitions of $\mathscr{H}_{i}, i=1,2,3$, the above is also equivalent with (3.17).

\section{A.4 Proof of Theorem 4.1}

Recall that $\mathcal{J}$ is the restriction of $J$ and is deterministic. For any $t \in[0, T)$, by (H5) and the DCT,

$$
J\left(\Phi^{\varepsilon} ; t, \overline{\mathscr{X}}(t)\right)-J(\bar{\Phi} ; t, \overline{\mathscr{X}}(t))=\left.\left[\mathcal{J}\left(\Phi^{\varepsilon} ; t, y\right)-\mathcal{J}(\bar{\Phi} ; t, y)\right]\right|_{y=\overline{\mathscr{X}}(t)} \cdot \text { a.s. }
$$


In the remaining part of the proof, let $\mathscr{X}^{\varepsilon}(\cdot), \overline{\mathscr{X}}(\cdot)$ be the solution of $(2.4)$ associated with $\left(\Phi^{\varepsilon}, t, y\right),(\bar{\Phi}, t, y)$, respectively. Using the same ideas as in Theorem 3.1, we obtain that

$$
\begin{aligned}
& \mathcal{J}\left(\Phi^{\varepsilon} ; t, y\right)-\mathcal{J}(\bar{\Phi} ; t, y) \\
& =\mathbb{E} \int_{t}^{t+\varepsilon}\left[\delta g\left(t, s, y, \mathscr{X}^{\varepsilon}(s)\right)+\left[\mathbb{E}_{t} H^{\prime}(t, \varepsilon)\right] \delta \mathscr{G}_{1}\left(s, \mathscr{X}^{\varepsilon}(s)\right)\right. \\
& \left.\quad+\delta \mathscr{G}_{2}\left(t, s, y, \mathscr{X}^{\varepsilon}(s)\right)\right] d s \equiv \sum_{i=1}^{3} \mathcal{R}_{i}(t, \varepsilon), \\
& H^{\prime}(t, \varepsilon):=\left.\int_{0}^{1} \partial_{z} h\left(t, y, \mathscr{X}^{\varepsilon}(T), z\right)\right|_{z=(1-\theta) \mathbb{E}_{t}} \overline{\mathscr{X}}_{(T)+\theta \mathbb{E}_{t} \mathscr{X}^{\varepsilon}(T)} d \theta .
\end{aligned}
$$

Observe that $\mathbb{E}_{t} H^{\prime}(t, \varepsilon)=\mathbb{E} H^{\prime}(t, \varepsilon)$ and $\mathscr{X}^{\varepsilon}(r)=X_{1}(r), r \in[t, t+\varepsilon)$, where $X_{1}(\cdot) \equiv y$ on $[0, t]$, and

$$
X_{1}(r)=y+\int_{t}^{r} b^{\Phi^{\prime}}\left(s, X_{1}(s)\right) d s+\int_{t}^{r} \sigma^{\Phi^{\prime}}\left(s, X_{1}(s)\right) d W(s), \quad r \in[t, T] .
$$

Since $\bar{\Phi}, \Phi^{\prime} \in \mathscr{D}_{a d}$, for any $k>2$, there exists constant $K$ such that

$$
\left\{\begin{array}{l}
\mathbb{E} \sup _{r \in[t, t+\varepsilon]}\left|X_{1}(r)-y\right|^{k} \leq K \cdot \varepsilon^{\frac{k}{2}-1} \mathbb{E} \int_{t}^{t+\varepsilon}\left[1+\left|X_{1}(s)\right|\right]^{k} d s, \\
\mathbb{E} \sup _{r \in[t, t+\varepsilon]}|\overline{\mathscr{X}}(r)-y|^{k} \leq K \cdot \varepsilon^{\frac{k}{2}-1} \mathbb{E} \int_{t}^{t+\varepsilon}[1+|\overline{\mathscr{X}}(s)|]^{k} d s .
\end{array}\right.
$$

For the term involving $\delta g$ in (A.12), let us denote it by $\mathcal{R}_{1}(t, \varepsilon)$. Since $\Phi^{\prime} \in \mathscr{D}_{a d}, \bar{\Phi} \in \mathscr{D}_{a d}$, $\delta g\left(t, \cdot, y, X_{1}(\cdot)\right)$ is right-continuous in $[t, t+\varepsilon]$, and

$$
\begin{gathered}
\lim _{\varepsilon \downarrow 0} \sup _{s \in[t, t+\varepsilon]}\left|\delta g\left(t, s, y, X_{1}(s)\right)-\delta g(t, t, y, y)\right|=0 . \text { a.s. } \\
\mathbb{E} \sup _{s \in[t, t+\varepsilon]}\left|\delta g\left(t, s, y, X_{1}(s)\right)-\delta g(t, t, y, y)\right|<\infty \text {. a.s. }
\end{gathered}
$$

Using the DCT, we see that

$$
\lim _{\varepsilon \rightarrow 0} \frac{\mathcal{R}_{1}(t, \varepsilon)}{\varepsilon}=g^{\Phi^{\prime}}(t, t, y, y)-g^{\bar{\Phi}}(t, t, y, y)
$$

We continue to look at the term involving $H^{\prime}(t, \varepsilon)$ on the right hand of (A.12),

$$
\mathcal{R}_{2}(t, \varepsilon):=\mathbb{E} H^{\prime}(t, \varepsilon) \mathbb{E} \int_{t}^{t+\varepsilon} \delta \mathscr{G}_{1}\left(r, X_{1}(r)\right) d r
$$

where for $r \in[t, t+\varepsilon], \delta \mathscr{G}_{1}(r, x)$ is defined in (3.3). We first treat $H^{\prime}(t, \varepsilon)$. From the polynomial growth condition of $\partial_{y} h$ in (H5), there exists $k>2$ and $K>0$ such that

$$
\left|H^{\prime}(t, \varepsilon)\right| \leq K\left[1+|y|^{k}+|\overline{\mathscr{X}}(T)|^{k}+\left|\mathscr{X}^{\varepsilon}(T)-\overline{\mathscr{X}}(T)\right|^{k}\right] .
$$

Since $\bar{\Phi} \in \mathscr{D}_{\text {ad }}$, we have $x \mapsto b^{\bar{\Phi}}(s, x), x \mapsto \sigma^{\bar{\Phi}}(s, x)$ are Lipschitz, linear growth, and

$$
\mathbb{E}\left|\mathscr{X}^{\varepsilon}(T)-\overline{\mathscr{X}}(T)\right|^{k} \leq K \mathbb{E}\left|\mathscr{X}^{\varepsilon}(t+\varepsilon)-\overline{\mathscr{X}}(t+\varepsilon)\right|^{k} \leq K \varepsilon^{\frac{k}{2}-1}, \quad k>2,
$$


where the second inequality comes from (A.13). By the continuity of $(x, y) \mapsto \partial_{y} h(t, \gamma, x, y)$, the DCT, and the polynomial growth of $\partial_{y} \Pi$,

$$
\lim _{\varepsilon \rightarrow 0} \mathbb{E} H^{\prime}(t, \varepsilon)=\mathbb{E}\left[\left.\partial_{z} h(t, y, \overline{\mathscr{X}}(T), z)\right|_{z=\mathbb{E}_{t}[\overline{\mathscr{X}}(T)]}\right]=\bar{\Pi}(t, t, y, y) .
$$

Since $\Phi^{\prime}, \Phi \in \mathscr{D}_{a d}$, we have that $b^{\Phi^{\prime}}, \sigma^{\Phi^{\prime}}, b^{\bar{\Phi}}, \sigma^{\bar{\Phi}}$ are linear growth. Using the polynomial growth condition of $\partial_{x} \bar{\Lambda}, \partial_{x x} \bar{\Lambda}$, there exists $k \in \mathbb{N}$ such that

$$
\sup _{s \in[t, t+\varepsilon]}\left|\delta \mathscr{G}_{1}\left(s, X_{1}(s)\right)\right| \leq K\left[1+\sup _{s \in[t, T]}\left|X_{1}(s)\right|^{k}\right] .
$$

By $(\mathrm{H} 5)$ and $\Phi^{\prime} \in \mathscr{D}_{a d}$, we observe that $\left.\bar{\Lambda} \in C^{1,2}, t \mapsto b^{\Phi^{\prime}}(t, x), \sigma^{\Phi^{\prime}}(t, x), b^{\bar{\Phi}}(t, x)\right), \sigma^{\bar{\Phi}}(t, x)$ are right-continuous for fixed $x \in \mathbb{R}^{n}$, and thus

$$
\lim _{\varepsilon \downarrow 0} \sup _{s \in[t, t+\varepsilon]}\left|\delta \mathscr{G}_{1}\left(s, X_{1}(s)\right)-\delta \mathscr{G}_{1}(t, y)\right|=0 .
$$

Hence by the DCT, $\lim _{\varepsilon \rightarrow 0} \mathbb{E} \int_{t}^{t+\varepsilon} \frac{\delta \mathscr{G}_{1}\left(r, X_{1}(r)\right)}{\varepsilon} d r=\delta \mathscr{G}_{1}(t, y)$. To summarize the above arguments, recalling (3.2),

$$
\lim _{\varepsilon \rightarrow 0} \frac{\mathcal{R}_{2}(t, \varepsilon)}{\varepsilon}=\bar{\Pi}(t, t, y, y)\left[(\mathscr{A} \bar{\Lambda}(t, \cdot))^{\Phi^{\prime}}(y)-(\mathscr{A} \bar{\Lambda}(t, \cdot))^{\bar{\Phi}}(y)\right]
$$

We treat the remaining term in $(\mathrm{A} .12)$ with $\delta \mathscr{G}_{2}(t, \cdot, y, \cdot)$ in $(3.3)$. From the polynomial growth of $\partial_{y} \bar{\Xi}, \partial_{y y} \bar{\Xi}$, the linear growth of $b^{\bar{\Phi}}, \sigma^{\bar{\Phi}}$, for some $k \geq 2$,

$$
\mathbb{E}\left[\sup _{s \in[t, T]}\left|\delta \mathscr{G}_{2}\left(t, r, y, X_{1}(r)\right)\right|\right] \leq K \mathbb{E}\left[\sup _{s \in[t, T]}\left(1+\left|X_{1}(s)\right|^{k}\right)+|y|^{k}\right]<\infty \text {. a.s. }
$$

Due to $\bar{\Phi}^{\prime} \in \mathscr{D}_{a d}$ and (H5), for fixed $t \in[0, T], x \in \mathbb{R}^{n}$, we see that $\partial_{z z} \bar{\Xi}(t, \cdot, x, \cdot), \partial_{z} \bar{\Xi}(t, \cdot, x, \cdot)$, $b^{\Phi^{\prime}}(\cdot, x), \sigma^{\Phi^{\prime}}(\cdot, x)$ are right-continuous at point $t$ and thus

$$
\lim _{\varepsilon \rightarrow 0} \sup _{s \in[t, t+\varepsilon]}\left|\delta \mathscr{G}_{2}\left(t, r, y, X_{1}(r)\right)-\delta \mathscr{G}_{2}(t, t, y, y)\right|=0 . \quad \text { a.s. }
$$

Hence by the DCT,

$$
\lim _{\varepsilon \rightarrow 0} \mathbb{E} \int_{t}^{t+\varepsilon} \frac{\delta \mathscr{G}_{2}\left(t, r, y, X_{1}(r)\right)}{\varepsilon} d r=\delta \mathscr{G}_{2}(t, t, y, y) .
$$

To sum up the above arguments, from (A.11), (A.12), (A.14), (A.17), (A.18), we obtain that $\bar{\Phi}$ is closed-loop equilibrium strategy if and only if for any $t \in[0, T)$,

$$
\begin{aligned}
& g^{\Phi^{\prime}}(t, t, y, y)-g^{\bar{\Phi}}(t, t, y, y)+\bar{\Pi}(t, t, y, y)\left[(\mathscr{A} \bar{\Lambda}(t, \cdot))^{\Phi^{\prime}}(y)-(\mathscr{A} \bar{\Lambda}(t, \cdot))^{\bar{\Phi}}(y)\right] \\
& +(\mathscr{A} \bar{\Xi}(t, t, y, \cdot))^{\Phi^{\prime}}(y)-(\mathscr{A} \bar{\Xi}(t, t, y, \cdot))^{\bar{\Phi}}(y) \geq 0 .
\end{aligned}
$$

Therefore, it is easy to see the above (4.8). 


\section{A.5 Proof of Theorem 4.2}

First under (H6), we point out that (H2) holds associated with the given $\bar{\Phi}$ and any $\Phi^{\prime} \in \mathscr{D}_{a d}^{0}$. From (3.6), we have

$$
\begin{aligned}
& J\left(\Phi^{\varepsilon} ; t, \overline{\mathscr{X}}(t)\right)-J(\bar{\Phi} ; t, \overline{\mathscr{X}}(t)) \\
& =\mathbb{E}_{t} \int_{t}^{t+\varepsilon}\left[\delta g\left(t, s, \overline{\mathscr{X}}(t), \mathscr{X}^{\varepsilon}(s)\right)+\left[\mathbb{E}_{t} H(t, \varepsilon)\right] \delta \mathscr{G}_{1}\left(s, \mathscr{X}^{\varepsilon}(s)\right)\right. \\
& \left.\quad+\delta \mathscr{G}_{2}\left(t, s, \overline{\mathscr{X}}(t), \mathscr{X}^{\varepsilon}(s)\right)\right] d s \equiv \sum_{i=1}^{3} \mathcal{R}_{i}(t, \varepsilon) .
\end{aligned}
$$

As to $\mathcal{R}_{1}(t, \varepsilon)$, we observe that for $s \in[t, t+\varepsilon]$,

$$
\begin{aligned}
& \left|g^{\Phi^{\prime}}\left(t, s, \overline{\mathscr{X}}(t), X_{1}(s)\right)-g^{\Phi^{\prime}}(s, s, \overline{\mathscr{X}}(s), \overline{\mathscr{X}}(s))\right| \\
& \leq K \sup _{s \in[t, t+\varepsilon]}\left[1+|\overline{\mathscr{X}}(t)|+|\overline{\mathscr{X}}(s)|+\left|X_{1}(s)\right|\right]^{k}\left[\left|X_{1}(s)-\overline{\mathscr{X}}(s)\right|+\varepsilon+|\overline{\mathscr{X}}(t)-\overline{\mathscr{X}}(s)|\right] .
\end{aligned}
$$

Using (A.13) with $\mathbb{E}, y$ replaced by $\mathbb{E}_{t}, \overline{\mathscr{X}}(t)$, for $k>2$, and any $\left\{\varepsilon_{n}\right\}$ tending to 0 , one has

$$
\mathbb{E}_{t} \int_{t}^{t+\varepsilon_{n}} \frac{\left|g^{\Phi^{\prime}}\left(t, s, \overline{\mathscr{X}}(t), X_{1}(s)\right)-g^{\Phi^{\prime}}(s, s, \overline{\mathscr{X}}(s), \overline{\mathscr{X}}(s))\right|}{\varepsilon_{n}} d s \rightarrow 0 .
$$

Similarly, with $k>2$, as $\varepsilon_{n} \rightarrow 0$,

$$
\mathbb{E}_{t} \int_{t}^{t+\varepsilon_{n}} \frac{\left|g^{\bar{\Phi}}\left(t, s, \overline{\mathscr{X}}(t), X_{1}(s)\right)-g^{\bar{\Phi}}(s, s, \overline{\mathscr{X}}(s), \overline{\mathscr{X}}(s))\right|}{\varepsilon_{n}} d s \rightarrow 0 .
$$

Therefore, we see that

$$
\lim _{\varepsilon_{n} \rightarrow 0} \frac{\mathcal{R}_{1}\left(t, \varepsilon_{n}\right)}{\varepsilon_{n}}=\lim _{\varepsilon_{n} \rightarrow 0} \mathbb{E}_{t} \int_{t}^{t+\varepsilon_{n}} \frac{\delta g(s, s, \overline{\mathscr{X}}(s), \overline{\mathscr{X}}(s))}{\varepsilon_{n}} d s .
$$

To treat $\mathcal{R}_{2}\left(t, \varepsilon_{n}\right)$, we first look at $\delta \mathscr{G}_{1}(\cdot, \cdot)$. By $(\mathrm{H} 6)$, for $s \in\left[t, t+\varepsilon_{n}\right]$,

$$
\begin{aligned}
& \left|\delta \mathscr{G}_{1}\left(s, X_{1}(s)\right)-\delta \mathscr{G}_{1}(s, \overline{\mathscr{X}}(s))\right| \\
& \leq K \sup _{s \in\left[t, t+\varepsilon_{n}\right]}\left[1+|\overline{\mathscr{X}}(s)|+\left|X_{1}(s)\right|\right]^{k}\left[\left|X_{1}(s)-\overline{\mathscr{X}}(t)\right|+|\overline{\mathscr{X}}(t)-\overline{\mathscr{X}}(s)|\right],
\end{aligned}
$$

from which

$$
\lim _{\varepsilon_{n} \rightarrow 0} \mathbb{E}_{t} \int_{t}^{t+\varepsilon_{n}} \frac{\delta \mathscr{G}_{1}\left(s, X_{1}(s)\right)}{\varepsilon_{n}} d s=\lim _{\varepsilon_{n} \rightarrow 0} \mathbb{E}_{t} \int_{t}^{t+\varepsilon_{n}} \frac{\delta \mathscr{G}_{1}(s, \overline{\mathscr{X}}(s))}{\varepsilon_{n}} d s .
$$

Similar as the above (A.15), we also have

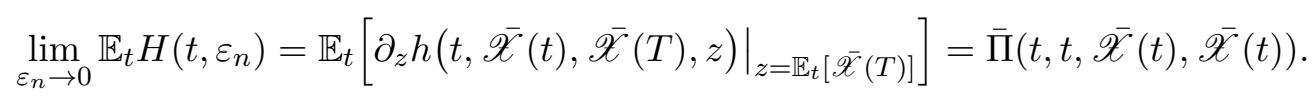

By the continuity of $\overline{\mathscr{X}}(\cdot)$ and the requirements of $\bar{\Pi}$ in $(\mathrm{H} 6)$, we have

$$
\lim _{\varepsilon_{n} \rightarrow 0} \mathbb{E}_{t} \sup _{s \in\left[t, t+\varepsilon_{n}\right]}|\bar{\Pi}(t, t, \overline{\mathscr{X}}(t), \overline{\mathscr{X}}(t))-\bar{\Pi}(s, s, \overline{\mathscr{X}}(s), \overline{\mathscr{X}}(s))|^{2}=0 .
$$


From the above (A.16), there exists $k \in \mathbb{N}$ such that

$$
\mathbb{E}_{t} \sup _{s \in\left[t, t+\varepsilon_{n}\right]}\left|\delta \mathscr{G}_{1}(s, \overline{\mathscr{X}}(s))\right|^{2} \leq K\left[1+\mathbb{E}_{t} \sup _{s \in[t, T]}|\overline{\mathscr{X}}(s)|^{k}\right]<\infty \text {. a.s. }
$$

As a result,

$$
\begin{aligned}
& \bar{\Pi}(t, t, \overline{\mathscr{X}}(t), \overline{\mathscr{X}}(t)) \lim _{\varepsilon_{n} \rightarrow 0} \mathbb{E}_{t} \int_{t}^{t+\varepsilon_{n}} \frac{\delta \mathscr{G}_{1}(s, \overline{\mathscr{X}}(s))}{\varepsilon_{n}} d s \\
& =\lim _{\varepsilon_{n} \rightarrow 0} \mathbb{E}_{t} \int_{t}^{t+\varepsilon_{n}} \frac{\bar{\Pi}(s, s, \overline{\mathscr{X}}(s), \overline{\mathscr{X}}(s)) \delta \mathscr{G}_{1}(s, \overline{\mathscr{X}}(s))}{\varepsilon_{n}} d s .
\end{aligned}
$$

To treat $\mathcal{R}_{3}\left(t, \varepsilon_{n}\right)$, it follows from the assumptions in (H6) that

$$
\begin{aligned}
& \left|\delta \mathscr{G}_{2}\left(t, s, \overline{\mathscr{X}}(t), X_{1}(s)\right)-\delta \mathscr{G}_{2}(s, s, \overline{\mathscr{X}}(s), \overline{\mathscr{X}}(s))\right| \\
& \leq K\left[1+|\overline{\mathscr{X}}(t)|+|\overline{\mathscr{X}}(s)|+\left|X_{1}(s)\right|\right]^{k}\left[\left|X_{1}(s)-\overline{\mathscr{X}}(s)\right|+\varepsilon_{n}+|\overline{\mathscr{X}}(t)-\overline{\mathscr{X}}(s)|\right] .
\end{aligned}
$$

Consequently, we arrive at

$$
\lim _{\varepsilon_{n} \rightarrow 0} \frac{\mathcal{R}_{3}\left(t, \varepsilon_{n}\right)}{\varepsilon_{n}}=\lim _{\varepsilon_{n} \rightarrow 0} \mathbb{E}_{t} \int_{t}^{t+\varepsilon_{n}} \frac{\delta \mathscr{G}_{2}(s, s, \overline{\mathscr{X}}(s), \overline{\mathscr{X}}(s))}{\varepsilon_{n}} d s
$$

To summarize, by (A.19), (A.20), (A.21), (A.22), (A.23), we see that

$$
\begin{aligned}
& \lim _{\varepsilon_{n} \rightarrow 0} \frac{J\left(\Phi^{\varepsilon_{n}} ; t, \overline{\mathscr{X}}(t)\right)-J(\bar{\Phi} ; t, \overline{\mathscr{X}}(t))}{\varepsilon_{n}} \\
& =\lim _{\varepsilon_{n} \rightarrow 0} \mathbb{E}_{t} \int_{t}^{t+\varepsilon_{n}} \frac{H_{d}\left(s, \overline{\mathscr{X}}(s), \Phi^{\prime}(s, \overline{\mathscr{X}}(s))\right)-H_{d}(s, \overline{\mathscr{X}}(s), \bar{\Phi}(s, \overline{\mathscr{X}}(s)))}{\varepsilon_{n}} d s
\end{aligned}
$$

On the other hand, by the integrability of $\overline{\mathscr{X}}(\cdot)$ and $(\mathrm{H} 6)$,

$$
\mathbb{E} \int_{0}^{T}\left|H_{d}\left(s, \overline{\mathscr{X}}(s), \Phi^{\prime}(s, \overline{\mathscr{X}}(s))\right)-H_{d}(s, \overline{\mathscr{X}}(s), \bar{\Phi}(s, \overline{\mathscr{X}}(s)))\right|^{2} d s<\infty .
$$

Using the Lemma 3.5 of [25], for almost all $t \in[0, T]$, there exists a subsequence, still denoted by $\left\{\varepsilon_{n}\right\}$, which may depend on $t$, such that

$$
\begin{aligned}
& \lim _{\varepsilon_{n} \rightarrow 0} \mathbb{E}_{t} \int_{t}^{t+\varepsilon_{n}} \frac{H_{d}\left(s, \overline{\mathscr{X}}(s), \Phi^{\prime}(s, \overline{\mathscr{X}}(s))\right)-H_{d}(s, \overline{\mathscr{X}}(s), \bar{\Phi}(s, \overline{\mathscr{X}}(s)))}{\varepsilon_{n}} d s \\
& =H_{d}\left(t, \overline{\mathscr{X}}(t), \Phi^{\prime}(t, \overline{\mathscr{X}}(t))\right)-H_{d}(t, \overline{\mathscr{X}}(t), \bar{\Phi}(t, \overline{\mathscr{X}}(t)))
\end{aligned}
$$

To sum up the above arguments, if $\bar{\Phi}$ is a closed-loop equilibrium strategy, then we obtain (4.8) for almost all $t \in[0, T]$. Conversely, if (4.8) for almost all $t \in[0, T]$, then it follows from (A.24) that $\bar{\Phi}$ is a closed-loop equilibrium strategy.

\section{References}

[1] S. Basak and G. Chabakauri, Dynamic mean-variance asset allocation, Rev. Finan. Stud. 23 (2010) 2970-3016. 
[2] T. Björk, M. Khapko and A. Murgoci, On time-inconsistent stochastic control in continuous time, Finance Stoch 21 (2017) 331-360.

[3] T. Björk, A. Murgoci and X. Zhou, Mean-variance portfolio optimization with state-dependent risk aversion, Math. Finance 24 (2014) 1-24.

[4] I. Ekeland and A. Lazrak, The golden rule when preferences are time inconsistent, Math. Financ. Econ. 4 (2010) 29-55.

[5] I. Ekeland and T. Pirvu, Investment and consumption without commitment, Math. Financ. Econ. 2 (2008) 57-86.

[6] I. Ekeland, O. Mbodji and T. Pirvu, Time-consistent portfolio management, SIAM J. Financial Math. 3 (2012) 1-32.

[7] K. Eliaz and R. Splieger, Contracting with diversely naive agents, Rev. Econ. Stud. 73 (2006) 689-714.

[8] W. Fleming and R. Rishel, Deterministic and stochastic optimal control, Springer-Verlag, 1975.

[9] J. Gu, S. Si and H. Zheng, Constrained utility deviation-risk optimization and time-consistent HJB equation, SIAM J. Control Optim. 58 (2020) 866-894.

[10] X. He, S. Hu, J. Oblój and X. Zhou, Optimal exit time from casino gambling: strategies of precommitted and naive gamblers, SIAM J. Control Optim. 57 (2019) 1845-1868.

[11] X. He and Z. Jiang, On the equilibrium strategies for time-inconsistent problems in continuous time, working paper, ssrn:3308274.

[12] C. Hernández and D. Possamai, Me, myself and I: a general theory of non-Markovian timeinconsistent stochastic control for sophisticated agents, working paper, arXiv:2002.12572.

[13] Y. Hu, J. Ma and J. Yong, On semi-linear degenerate backward stochastic partial differential equations, Probab. Theory Relat. Fields 123 (2002) 381-411.

[14] J. Huang, X. Li and T. Wang, Characterizations of closed-loop equilibrium solutions for dynamic mean-variance optimization problems, Syst. Control Lett. 110 (2017) 15-20.

[15] Y. Huang, A. Nguyen-Huu and X. Zhou, ' General stopping behaviors of naive and noncommitted sophisticated agents, with application to probability distortion. Math Finance 30 (2020) $310-340$.

[16] D. Hume, A treatise of human nature, First edition, 1739; Reprint, Oxford Univ. Press, 1978.

[17] K. Lindensjö, A regular equilibrium solves the extended HJB system, Oper. Res. Lett. 47 (2019) 427-432.

[18] J. Marin-Solano and J. Navas, Consumption and portfolio rules for time inconsistent investors, Euro. J. Oper. Res. 201 (2010) 860-872.

[19] I. Palacios-Huerta, Time-inconsistent preferences in Adam Smith and Davis Hume, Histroy Pol. Econ. 35 (2003) 241-268.

[20] A. Smith, The theory of moral sentiments, First edition, 1759; Reprint, Oxford Univ. Press, 1976. 
[21] R. Strotz, Myopia and inconsistency indynamic utility maximization, Rev. Econ. Stud. 23 (1955) 165-180.

[22] X. Su, T. Wang, J. Wei and C. Zhou, Dynamic mean-variance portfolio selection problems with random coefficients, working paper.

[23] H. Wang and Z. Wu, Time-inconsistent optimal control problem with random coefficients and stochastic equilibrium HJB equation, Math. Control Related Fields 5 (2015) 651-678.

[24] T. Wang, Equilibrium controls in time inconsistent stochastic linear quadratic problems, Appl. Math. Optim. 81 (2020) 591-619.

[25] T. Wang, Necessary conditions in stochastic linear quadratic problems and their applications, J. Math. Anal. Appl. 469 (2019) 280-297.

[26] T. Wang and J. Yong, Backward stochastic Volterra integral equations-representation of adapted solutions, Stochastic Process Appl. 129 (2019) 4926-4964.

[27] K. Wong, S. Yam and H. Zheng, Utility-deviation-risk portfolio selection, SIAM J. Control Optim. 55 (2017) 1819-1861.

[28] J. Yong, Time-inconsistent optimal control problem and the equilibrium HJB equation, Math. Control Related Fields 2 (2012) 271-329.

[29] J. Yong, Time-inconsistent optimal control problems, in Proceedings of 2014 ICM, Section 16. Control Theory and Optimization, (2014) 947-969. 